\title{
THE EVOLUTION OF THE PECULIAR TYPE IA SUPERNOVA SN 2005HK OVER 400 DAYS
}

\author{
D.K. Sahu ${ }^{1}$, Masaomi Tanaka ${ }^{2}$, G.C. Anupama ${ }^{1}$, Koji S. Kawabata ${ }^{3}$, Keilchi Maeda ${ }^{4,5}$, Nozomu Tominaga $^{2}$, \\ Ken'ichi Nomoto ${ }^{2,5,6}$, PaOlo A. Mazzali ${ }^{4,6,7,8}$, And T.P.Prabhu ${ }^{1}$ \\ Accepted for publication in The Astrophysical Journal
}

\begin{abstract}
$U B V R I$ photometry and medium resolution optical spectroscopy of peculiar Type Ia supernova SN 2005hk are presented and analysed, covering the pre-maximum phase to around 400 days after explosion. The supernova is found to be underluminous compared to "normal" Type Ia supernovae. The photometric and spectroscopic evolution of SN 2005hk is remarkably similar to the peculiar Type Ia event SN 2002cx. The expansion velocity of the supernova ejecta is found to be lower than normal Type Ia events. The spectra obtained $\gtrsim 200$ days since explosion do not show the presence of forbidden [Fe II], [Fe III] and [Co III] lines, but are dominated by narrow, permitted Fe II, NIR $\mathrm{Ca}$ II and Na I lines with P-Cygni profiles. Thermonuclear explosion model with Chandrasekhar mass ejecta and a kinetic energy smaller $\left(E_{\mathrm{K}}=0.3 \times 10^{51} \mathrm{ergs}\right)$ than that of canonical Type Ia supernovae is found to well explain the observed bolometric light curve. The mass of ${ }^{56} \mathrm{Ni}$ synthesized in this explosion is $0.18 M_{\odot}$. The early spectra are successfully modeled with this less energetic model with some modifications of the abundance distribution. The late spectrum is explained as a combination of a photospheric component and a nebular component.
\end{abstract}

Subject headings: supernovae: general — supernovae: individual (SN 2005hk)

\section{INTRODUCTION}

An impressive homogeneity in the light curves and peak luminosities make Type Ia supernovae (SNe Ia) good candidates in the determination of the extragalactic distance scale. Though a majority of the observed SNe Ia belong to the "normal" type (Branch et al. 1993), a number of studies indicate significant photometric as well as spectroscopic differences. For example, studies of nearby supernovae by $\mathrm{Li}$ et al. (2001) indicate that $64 \% \mathrm{SNe}$ Ia are "normal", while $20 \%$ are of the overluminous SN $1991 \mathrm{~T}$ type and $16 \%$ of the underluminous SN 1991bg type. The peak absolute luminosities of SNe Ia are well correlated with their immediate post-maximum decline rate, forming a photometric sequence from the luminous blue events with a relatively slow decline rate to the faster, red, subluminous events (Hamuy et al. 1996a,b; Phillips et al. 1999).

However, there are a few SNe Ia that are known to deviate from this relation, the most notable amongst them being SN 2002cx. This supernova was found to be underluminous, but had a light curve decline rate

Electronic address: dks@iiap.res.in, mtanaka@astron.s.utokyo.ac.jp

${ }^{1}$ Indian Institute of Astrophysics, II Block Koramangala, Bangalore 560034, India

2 Department of Astronomy, Graduate School of Science, University of Tokyo, Hongo 7-3-1, Bunkyo-ku, Tokyo 113-0033, Japan

${ }^{3}$ Hiroshima Astrophysical Science Center, Hiroshima University, 1-3-1 Kagamiyama, Higashi-Hiroshima, Hiroshima 739-8526, Japan

${ }^{4}$ Max-Planck Institut für Astrophysik, Karl-SchwarzschildStrasse 1, Postfach 1317, D-85741 Garching, Germany

${ }^{5}$ Institute for the Physics and Mathematics of the Universe, University of Tokyo, Kashiwa, Chiba 277-8582, Japan

${ }^{6}$ Research Center for the Early Universe, Graduate School of Science, University of Tokyo, Bunkyo-ku, Tokyo 113-0033, Japan

7 INAF-Osservatorio Astronomico di Trieste, via Tiepolo 11, I-34131, Trieste, Italy

8 Kavli Institute for Theoretical Physics, University of California, Santa Barbara, CA 93106
$\Delta \mathrm{m}_{15}(B)=1.29$, comparable to normal $\mathrm{SNe}$ (Li et al. 2003). The early phase ( $\lesssim 100$ days after explosion) spectra indicate line velocities lower by a factor of 2 compared to those of normal SNe Ia (Li et al. 2003; Branch et al. 2004). Furthermore, the late phase $(\sim 250$ days after explosion) spectra are also quite dissimilar compared to normal SNe Ia and possibly consist of P-Cygni profiles (Jha et al. 2006).

Interestingly, SN 2002cx is not a unique event. Jha et al. (2006) and Phillips et al. (2007) have shown that SN 2005hk shows photometric and spectroscopic behaviour almost identical to that of SN 2002cx. Further, Jha et al. (2006) have shown that SNe 2003gq and 2005P also show spectra similar to SN 2002cx. Spectropolarimetric observations of SN 2005hk at the early phases by Chornock et al. (2006) indicate low polarization levels, indicating that the peculiarities of SN 2002cx-like SNe do not result from an extreme asphericity. Based on the analyses of the early phase spectra of SN 2002cx, Branch et al. (2004) suggest that the observed lower line velocities are consistent with the deflagration models of explosion. Jha et al. (2006) report a possible detection of $\mathrm{O}$ I lines in the late phase spectra and suggest large scale mixing in the central region, which is also consistent with threedimensional (3D) deflagration models. Phillips et al. (2007) find that, qualitatively the observed light curves of SN 2005hk are in reasonable agreement with model calculations of a 3D deflagaration model that produces $\sim 0.2 M_{\odot}$ of ${ }^{56} \mathrm{Ni}$.

Understanding the nature of this class of $\mathrm{SNe}$ Ia is thus quite important for the study of homogeneity and heterogeneity of SNe Ia. It may provide a caution for the cosmological use of SNe Ia and a strong constraint to the explosion models.

We present in this paper the photometric and spectroscopic development of SN 2005hk over $\sim 400$ days since explosion. 


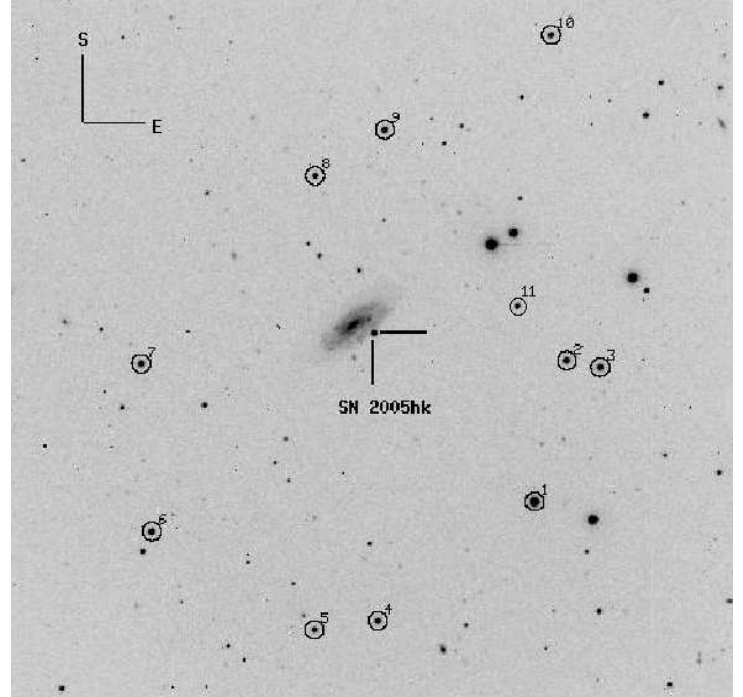

FiG. 1.- Identification chart for SN 2005hk. The stars used as local standards are marked as numbers 1-11.

\section{OBSERVATIONS AND DATA REDUCTION}

\subsection{Photometry}

Supernova SN 2005hk was observed in UBVRI bands with the $2 \mathrm{~m}$ Himalayan Chandra Telescope (HCT) of the Indian Astronomical Observatory (IAO), Hanle, India, using the Himalaya Faint Object Spectrograph Camera (HFOSC), equipped with a SITe $2 \mathrm{k}$ x $4 \mathrm{k}$ CCD. The central $2 \mathrm{k} \times 2 \mathrm{k}$ pixels were used for imaging which corresponds to a field of view of $10^{\prime} \times 10^{\prime}$, with a scale of 0.296 arcsec/pixel. The monitoring of the supernova started on 2005 November 5 (JD 2,453,680), $\sim 4$ days and $\sim 6$ days after its discovery by Burket \& Li (2005), and Barentine, et al. (2005), and continued until 2006 September 20 (JD 2,453,999). Standard fields PG0231+051, PG1047+003, PG0942-029, PG0918+027 (Landolt 1992) were observed on 2005 November 24 and 2005 December 28 under photometric conditions, and were used to calibrate a sequence of secondary standards in the supernova field.

The data reduction was done in the standard manner, using various tasks available within IRAF. The observed data were bias subtracted, flat-field corrected and cosmic-ray hits removed. Aperture photometry was performed on the standard stars, using an aperture radius determined using the aperture growth curve, and were calibrated using the average color terms and photometric zero points determined on the individual nights. The $U B V R I$ magnitudes of the secondary standards in the supernova field, calibrated and averaged over the two nights are listed in Table 1 . The secondary sequence is shown marked in Figure 1. The magnitudes of the supernova and the local standards were estimated using the profile fitting technique, with a fitting radius equal to the FWHM of the stellar profile. The difference between the aperture and profile-fitting magnitude was obtained using bright standards in the supernova field and this correction was applied to the supernova magnitude. The calibration of the supernova magnitude to the standard system was done differentially with respect to the local standards.

SN 2005hk was also observed on 2005 December 26, 2006 June 30 and November 27 with the Faint Object
Camera and Spectrograph (FOCAS; Kashikawa et al. 2002) mounted on the $8.2 \mathrm{~m}$ Subaru Telescope in the $B$ and $R$ bands. The local standard star 11 was calibrated as indicated in Table 1 using the HCT/HFOSC photometry of the star 2 in Figure 1, then the $B$ and $R$ magnitudes were derived by the point spread photometry in the DAOPHOT package of IRAF.

The observed supernova magnitudes are listed in Table 2 .

\subsection{Spectroscopy}

Spectroscopic monitoring of the supernova using the HCT began on 2005 November 4 and continued until 2006 January 12. The journal of observations is given in Table 3. The HCT spectra cover the wavelength ranges 3500-7000 $\AA$ and 5200-9100 $\AA$ at a spectral resolution of $\sim 7 \AA$. The data reduction was carried out in the standard manner using the tasks available within IRAF. The data were bias corrected, flat-fielded and the one dimensional spectra extracted using the optimal extraction method. Spectra of FeAr and FeNe lamps were used for wavelength calibration. The instrumental response curves were obtained using spectrophotometric standards observed on the same night and the supernova spectra were brought to a relative flux scale. The flux calibrated spectra in the two regions were combined to a weighted mean to give the final spectrum on a relative flux scale. The spectra were brought to an absolute flux scale using zero points derived by comparing the observed flux in the spectra with the flux estimated using the photometric magnitudes.

Spectra during the late phases were obtained with the Subaru/FOCAS on 2005 December 26, 2006 June 30 and November 27. A 0.8 arcsec width slit was used with the B300 grism, which gave a wavelength coverage of 4700-9000 $\AA$ and a spectral resolution of $11 \AA$. The exposure times were $900 \mathrm{sec}, 1,800 \mathrm{sec}$ and 3,600 sec for 2005 Dec 26, 2006 Jun 30 and Nov 27, respectively. The instrumental response was corrected using data of a spectrophotometric standard star (GD 153 or BD+28d4211). The flux was then scaled to be consistent with the $\mathrm{R}$ band photometry.

\section{UBVRI LIGHT CURVES}

The early phase light curves (LCs) of SN 2005hk are discussed by Phillips et al. (2007) and Stanishev et al. (2006). We present here the LCs of this supernova during the early as well as the late phase (ref. Table 2). Figure 2 shows the LCs of SN 2005hk in the $U, B, V$, $R$ and $I$ bands, respectively. The epoch of maximum in each of these bands is estimated by a cubic spline fit to the points around maximum. The supernova reached a maximum in the $B$ band on JD 2453685.34 with magnitude of $15.91 \pm 0.03$. The maximum brightness in the $V, R$ and $I$ bands occured $\sim+4,+6.5$ and +8.5 days, relative to the maximum in $B$ band, respectively. The decline rate parameter in the $B$ band estimated using our data is $\Delta \mathrm{m}_{15}(B)=1.68 \pm 0.05$. Our estimates of the dates of maximum, apparent maximum magnitudes and the decline rate parameter are listed in Table 4 . These estimates are consistent with the values reported by Phillips et al. (2007) and Stanishev et al. (2006).

The LCs of SN 2005hk are compared with those of the normal SNe 1994D $\left[\Delta m_{15}(B)=1.31 \pm 0.08\right.$; Richmond et 


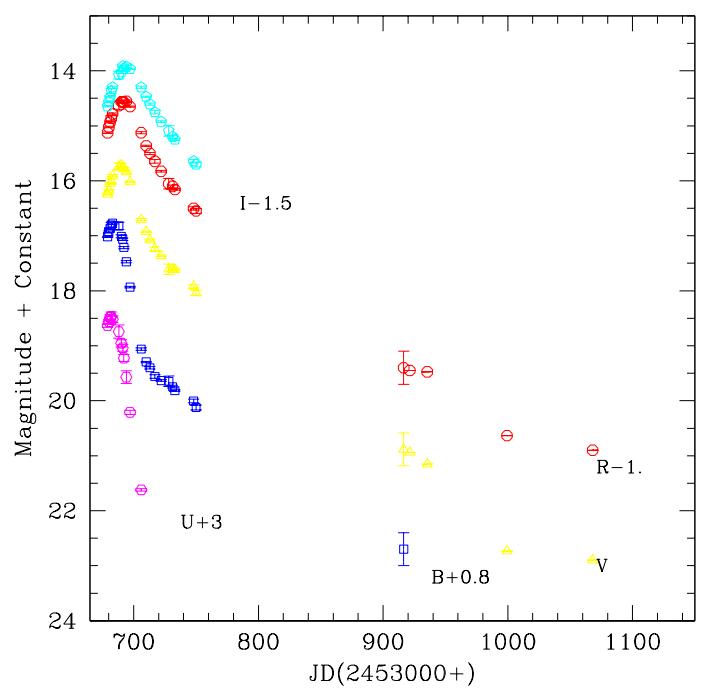

FIG. 2.- The $U B V R I$ LCs of SN 2005hk. The LCs have been shifted by the amount indicated in the legend.

al. 1995] and $2003 \mathrm{du}\left[\Delta m_{15} B=1.04 \pm 0.04\right.$; Anupama et al. 2005], the overluminous SN 1991T $\left[\Delta m_{15}(B)=\right.$ $0.95 \pm 0.05$; Lira et al. 1998], the subluminous SN 1991bg $\left[\Delta m_{15}(B)=1.93 \pm 0.08\right.$; Filippenko et al. 1992; Turatto et al. 1996] and the peculiar SN 2002cx $\left[\Delta m_{15}(B)=\right.$ $1.29 \pm 0.011 ; \mathrm{Li}$ et al. 2003, $\Delta m_{15}(B)=1.7 \pm 0.1$; Phillips et al. 2007] (Figures 3-4). The early LCs (Figure 3) indicate that the pre-maximum brightening of SN 2005hk was faster than the luminous SN $1991 \mathrm{~T}$ but comparable to the normal SNe 1994D and 2003du. Following the maximum, during the initial 20 days, the decline in $B$ was faster than in normal $\mathrm{SNe}$, but similar to $\mathrm{SN}$ $2002 \mathrm{cx}$, while the decline in $V$ was similar to the normal SN 1994D. Subsequently, beyond 30 days past maximum, the decline in $B$ and $V$ was slower than all the other SNe. The secondary peak seen in the $R$ and $I$ bands of SNe Ia was found to be absent in SN 2005hk, similar to the subluminous SN 1991bg. However, beyond $\sim 50$ days past maximum the $R$ LC of SN 2005hk had a decline similar to SN 1991T, which is slower than that seen in the normal and subluminous SNe (Figure 4). The evolution of the $U$ light curve of SN 2005hk was found to be faster than both the normal and the luminous types.

The early phase evolution of SN 2005hk is very similar to that of SN 2002cx. Both objects have similar $\Delta \mathrm{m}_{15}(B)$. SN $2005 \mathrm{hk}$ has a $\Delta \mathrm{m}_{15}(B)$ of 1.68 , while SN $2002 \mathrm{cx}$ has a value of 1.7 , as indicated by the the revised photometry by Phillips et al. (2007). On the other hand, at epochs beyond 15 days past maximum, SN 2005hk declines somewhat slower than SN 2002cx. The decline rate during $\sim 20-50$ days past $B$ maximum, in the $B$, $V, R$ and $I$ bands are, respectively, $0.021,0.027,0.031$ and $0.031 \mathrm{mag}$ day $^{-1}$, while the corresponding decline rates in SN 2002cx during the same phase are 0.037, $0.035,0.041$ and 0.035 . It may be noted here that no galaxy template subtraction is done for the data presented here, and the SN magnitudes may be affected by the host galaxy at the later phases. However, as suggested by Phillips et al.(2007), neither $K$ correction nor the galaxy background contamination is sufficient to explain the discrepancies seen in the LCs of SN 2005hk and SN 2002cx.

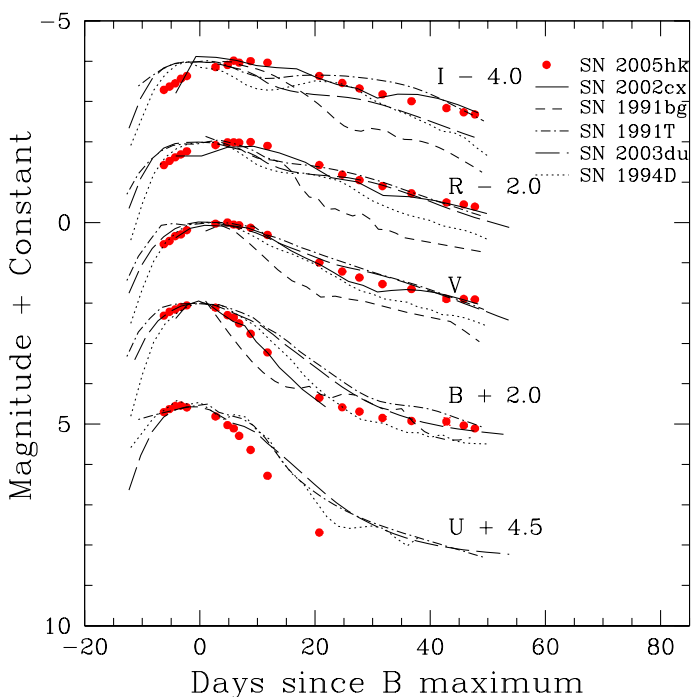

FIG. 3. - Early phase ( $<50$ days) LC of SN 2005 hk together with those of SNe 2002cx, 1991bg, 1991T, 2003du and 1994D. The ordinate in each panel is the magnitude below the respective maximum, and the abscissae represent the days since respective $B$ maximum. For clarity, the LCs have been shifted by the amount indicated in the legend.

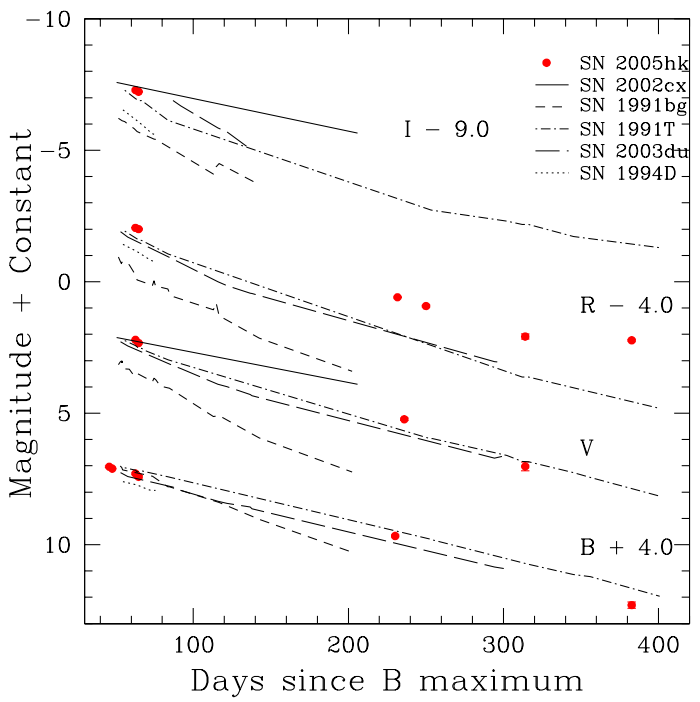

Fig. 4. - Late phase ( $>50$ days) LC of SN 2005hk together with those of SNe 2002cx, 1991bg, 1991T, 2003du and 1994D. The ordinate in each panel is the magnitude below the respective maximum, and the abscissae represent the days since respective $B$ maximum. For clarity, the LCs have been shifted by the amount indicated in the legend.

\section{REDDENING AND COLOR CURVES}

The reddening within our Galaxy in the direction of SN 2005hk is $E(B-V)_{\text {Gal }}=0.022$ (Schlegel et al. 1998). The reddening within the host galaxy may be estimated from the methods suggested by Phillips et al. (1999), Altavilla et al. (2004) and Lira (1996). The Phillips et al. (1999) and the Altavilla et al. (2004) methods give $E(B-V)_{\text {Host }}=0.20$ and 0.09 , respectively, while the Lira (1996) method indicates a higher value. Spectropolarimetric observations of SN 2005hk (Chornock et al. 2006) indicate an interstellar polarization of $0.27 \%$ produced by the host galaxy which, for the standard dust polar- 


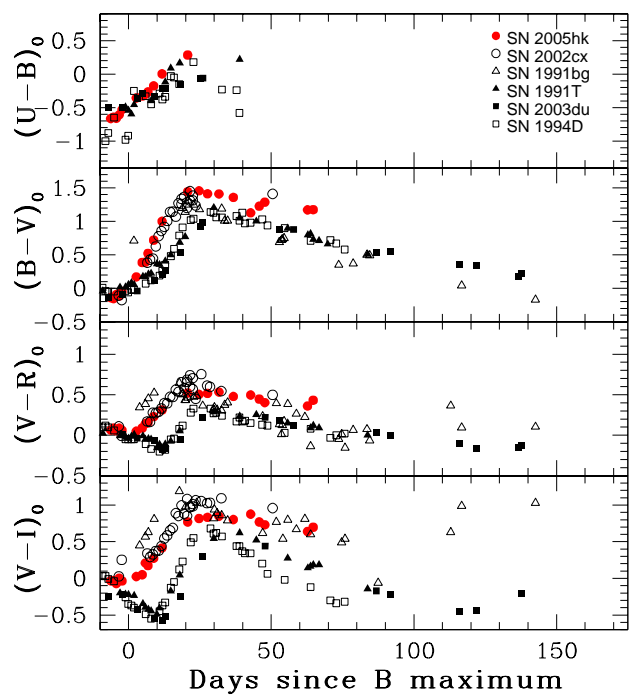

Fig. 5.- The $U-B, B-V, V-R$ and $V-I$ color curves of SN 2005hk. Also shown for comparison are the color curves of SNe 2002cx, 1991T, 1991bg, 2003du and 1994D. The abscissae correspond to days since respective $B$ maximum.

ization efficiency, corresponds to $E(B-V)_{\text {Host }}=0.09$. Owing to the peculiar nature of SN 2005hk, the methods based on normal SNe Ia may not be applicable. Hence, adopting the estimate for the host galaxy extinction as indicated by the spectropolarimetric observations, we estimate the total reddening towards SN 2005hk to be $E(B-V)_{\text {tot }}=0.11$.

The $(U-B),(B-V),(V-R)$ and $(V-I)$ color curves of SN 2005hk are shown in Figure 5, compared with other SNe. The color curves of all SNe have been corrected for reddening using the Cardelli et al. (1989) extinction law and the $E(B-V)$ values of $E(B-V)=$ 0.13 for SN 1991T (Phillips et al. 1992), $E(B-V)=$ 0.034 for SN 2002cx (Li et al. 2003), $E(B-V)=0.04$ for SN 1994D (Richmond et al. 1995), $E(B-V)=0.05$ for SN 1991bg (Turatto et al. 1996) and $E(B-V)=0.01$ for SN 2003du (Anupama et al. 2005).

The color curves of SN 2005hk are very similar to SN $2002 \mathrm{cx}$. The $(U-B)$ color evolution is very similar to SN 1991T. The $(B-V)$ and $(V-R)$ color evolution of SN 2005hk in the pre-maximum and maximum phase are very similar to the other SNe Ia except SN 1991bg, which had a considerably red color. Beyond $\sim 5$ days, until $\sim 20$ days after $B$ maximum, both $(B-V)$ and $(V-R)$ colors get increasingly redder compared to other SNe, but are still bluer compared to SN 1991bg. The $(V-I)$ color on the other hand gets redder than other $\mathrm{SNe} \sim 5$ days before maximum, and also does not show the dip seen in the color curves of other SNe Ia at $\sim 10$ days post-maximum. Beyond $\sim 20$ days after maximum, the $(V-R)$ and $(V-I)$ colors of SN 2005hk follow the trend of other supernovae and become marginally blue, while $(B-V)$ continues to be redder than other SNe, including SN 1991bg.

Interestingly, despite a very similar color evolution during the pre-maximum phase to $\sim 20$ days after $B$ maximum, SN 2005hk appears to be marginally bluer than SN 2002cx at later phases.

\section{BOLOMETRIC LIGHT CURVE}

\subsection{Behavior of bolometric light curve}

The bolometric LC of SN 2005hk is estimated using the $U B V R I$ photometry presented here along with the NIR $Y J H$ photometry reported by Phillips et al. (2007). The magnitudes were reddening corrected using the value estimated in the previous section and the Cardelli et al. (1989) extinction law. The corrected magnitudes were then converted to monochromatic fluxes using the zero points from Bessell et al. (1998). The bolometric fluxes were derived by fitting a spline curve to the $U, B, V, R, I, Y, J$ and $H$ fluxes and integrating over the wavelength range $3100 \AA$ to $1.63 \mu \mathrm{m}$. In the later phases when only $B, V, R$ or $V, R$ magnitudes were available, the bolometric flux were estimated by applying a bolometric correction to the available magnitudes. Since SN 2005hk belongs to a rare class of SNe Ia, the bolometric correction is derived from the light curve of SN 2005hk itself rather than assuming the corrections based on normal SNe Ia (Suntzeff 1996; Contrado et al. 2000). The bolometric corrections were estimated based on the last three points in the LC for which the $B V R I J H$ bolometric flux is available. A simple average of the bolometric corrections for these days give values of B.C=-0.926 \pm 0.089 in $B, B . C=0.294 \pm 0.045$ in $V$ and $B . C=0.677 \pm 0.027$ in $R$ band.

The bolometric LC is plotted in Figure 6 (red circles). Also plotted in the figure are the bolometric LCs of the normal (but slightly underluminous) Ia SN 1992A (black squares), the subluminous Ia SN 1991bg (blue triangles) and SN 2002cx (green triangles). The peak luminosity $\left(M_{b o l}=-17.7\right)$ is fainter than the canonical value for normal SNe Ia. It suggests that a smaller amount of ${ }^{56} \mathrm{Ni}$ is synthesized during the explosion.

The decline of the bolometric LC after the maximum is slower than that of SN 1992A although they have a similar maximum luminosity, as also shown by Phillips et al. (2007). Our observations extend the bolometric LC to $\sim 400$ days since explosion. At such late epochs, the bolometric luminosity of SN 2005hk is still brighter than that of SN 1992A. In addition, the difference in the luminosity between the two SNe becomes large, reaching $\sim 1$ magnitude at $>250$ days after the explosion. The bright late phase luminosity indicates a more efficient trapping of the $\gamma$-rays from decaying ${ }^{56} \mathrm{Co}$ in SN $2005 \mathrm{hk}$ compared to SN 1992A.

\subsection{Light curve modeling}

Phillips et al. (2007) suggest that qualitatively the observed properties of SN 2005hk are consistent with 3D deflagration model (e.g., Röpke et al. 2006, Blinnikov et al. 2006), showing the bolometric and multi-color LC model until $\sim 80$ days after the explosion. Branch et al. (2004) and Jha et al. (2006) also suggest a 3D deflagration model for SN 2002cx. Here we give the first investigation of the bolometric LC of this class of $\mathrm{SNe}$ until $\sim 400$ days after the explosion.

We use an LTE radiation transfer code (Iwamoto et al. 2000) for the computation of the bolometric LCs. For the $\gamma$-ray transport, a gray atmosphere is assumed. For optical radiation transport, electron-scattering and line opacity are taken into account. The electronscattering opacity is evaluated by solving the Saha equation while the line opacity is crudely assumed to be con- 


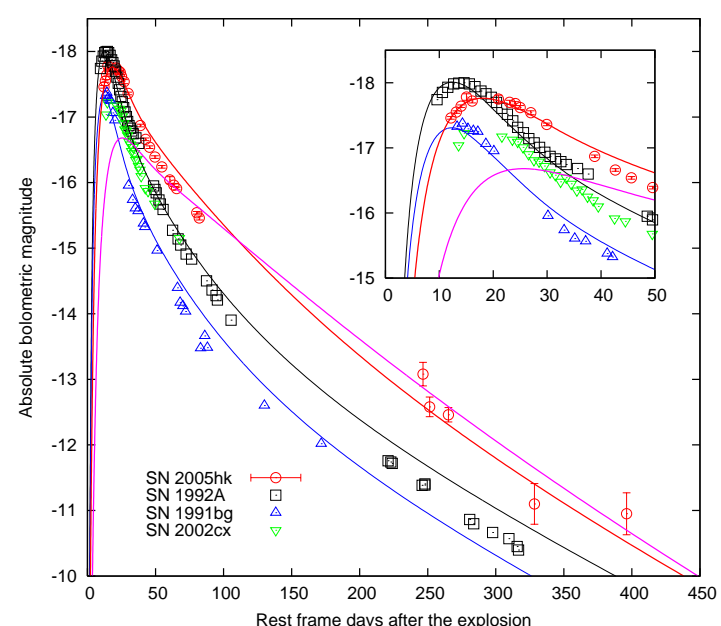

FIG. 6.- Bolometric LC of SN 2005hk (red circles) compared with a synthetic LC with the E03 model (red line). Black squares and blue triangles are bolometric LCs of SNe 1992A and 1991bg (Suntzeff 1996), respectively. Model LCs for these two SNe are shown in black and blue lines, respectively (based on W7 but different mass of ${ }^{56} \mathrm{Ni}$, see Table 5). Green triangles are the bolometric LC of SN 2002cx. In order to fix the rise time of the LCs of SNe $1992 \mathrm{~A}$ and 1991bg, $B$ band rise time is assumed as $t_{\text {rise }}=19.5 \times s$ days (Conley et al. 2006), where $s$ is stretch factor of $B$ band LC (Goldhaber et al. 2001). The $B$ band rise times of SNe 1992A $(s=0.80)$ and $1991 \mathrm{bg}(s=0.68)$ are estimated as 15.6 and 13.2 days, respectively. Since the LC of SN 2005hk is deviated from the normal sequence of SNe Ia, we simply assume $t_{\text {rise }}=17$ days as in Phillips et al. (2007). The magenta line shows the synthetic LC with the E008 model.

stant $\left(0.1 \mathrm{~cm}^{2} \mathrm{~g}^{-1}\right.$; Iwamoto 1997) for simplicity. We use the W7 deflagration model (Nomoto et al. 1984) as a standard density structure and abundance distributions.

First, the mass of ${ }^{56} \mathrm{Ni}\left[M\left({ }^{56} \mathrm{Ni}\right)\right]$ is varied from the $\mathrm{W} 7$ value $\left(M\left({ }^{56} \mathrm{Ni}\right)=0.59 M_{\odot}\right)$ to obtain a good agreement with the maximum luminosity of SNe Ia shown in Figure 6 . In this procedure, the total mass of iron group elements is kept constant to conserve the explosion energy. The black and blue lines in Figure 6 are the synthetic LCs with $M\left({ }^{56} \mathrm{Ni}\right)=0.17$ and $0.088 M_{\odot}$, respectively. Both at the early phase (ref. inset in Fig. 6) and the late phase, the LCs are in good agreement with SNe 1992A and 1991bg (Suntzeff 1996), respectively ${ }^{9}$. However, the model sequence with various $M\left({ }^{56} \mathrm{Ni}\right)$ never reproduces the brightness of SN 2005hk at $\gtrsim 50$ days after the explosion.

As also suggested by Phillips et al. (2007), we now try to explain the LC of SN 2005hk with less energetic models to fit the LC around maximum. We construct a less energetic model simply by scaling the structure of W7 homologously. The mass of the burned material should be adjusted accordingly by taking nuclear energy production into account. In our model, the mass fraction of each burned element is reduced by the same fraction $\left[\left(E_{\mathrm{K}}+E_{\mathrm{B}}\right) /\left(E_{\mathrm{W} 7}+E_{\mathrm{B}}\right)\right]$ in every radial shell. Here $E_{\mathrm{K}}$, $E_{\mathrm{B}}$ and $E_{\mathrm{W} 7}$ are the kinetic energy of the SN ejecta, the binding energy of a progenitor WD $\left(\sim 0.5 \times 10^{51}\right.$ ergs $)$, and the kinetic energy of W7 model $\left(\sim 1.3 \times 10^{51}\right.$ ergs), respectively. The mass of the burned elements reduced by the procedure above is compensated by unburned $\mathrm{C}$ and $\mathrm{O}$, with the mass fraction of $\mathrm{X}(\mathrm{C})=0.5$ and

\footnotetext{
9 This shows that our crude assumption of constant line opacity
} $\left(0.1 \mathrm{~cm}^{2} \mathrm{~g}^{-1}\right)$ is reasonable.

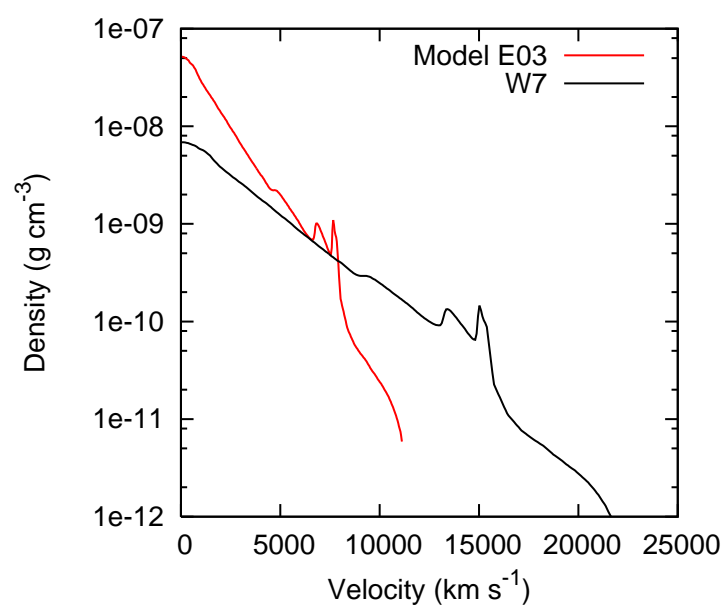

FIG. 7.- The density structure of model E03 compared with that of W7 (1 day after the explosion). The kinetic energy of model E03 is $E_{\mathrm{K}}=0.3 \times 10^{51} \mathrm{ergs}$. The density structure is scaled from that of W7 homologously. This makes the density at the inner part higher than the original.

$\mathrm{X}(\mathrm{O})=0.5$.

The red line in Figure 6 is the synthetic LC with $M\left({ }^{56} \mathrm{Ni}\right)=0.18 M_{\odot}$ and $E_{\mathrm{K}}=0.3 \times 10^{51} \mathrm{ergs}$. This kinetic energy is similar to that of the $3 \mathrm{D}$ deflagration model used in Phillips et al. (2007). The density structure of this model is given in Figure 7 together with that of the original W7. This model gives a reasonable fit to SN 2005hk at late phase as well as at early phase. In the figure, we assume $B$ maximum of SN $2005 \mathrm{hk}$ occurs 17 days after the explosion, which gives the best fit of the LC (see also Phillips et al. $2007^{10}$ ). The mass of ${ }^{56} \mathrm{Ni}$ derived from the model is somewhat smaller than $0.24 M_{\odot}$ derived by Phillips et al. (2007). It may be caused by a different distribution of ${ }^{56} \mathrm{Ni}$ in the ejecta. Since ${ }^{56} \mathrm{Ni}$ in the model in Phillips et al. (2007) is extended to the outer layers, a part of ${ }^{56} \mathrm{Ni}$ does not contribute to the optical light. In this paper, we call our scaled, less energetic model E03.

The behavior of the model E03 LC is understood by simple analysis. If the opacity is assumed to be constant, the time scale of the bolometric LC $\left(\tau_{L C}\right)$ is scaled as $\tau_{\mathrm{LC}} \propto M_{\mathrm{ej}}^{3 / 4} E_{\mathrm{K}}^{-1 / 4}$ (Arnett 1982), where $M_{\mathrm{ej}}$ is the mass of the ejecta. Therefore, if $M_{\mathrm{ej}}$ is fixed (Chandrasekhar mass), the lower energy gives a slow decline after the maximum. The late phase evolution of LC is mainly determined by the optical depth to $\gamma$-rays. Since the optical depth is higher for lower energetic ejecta $(\tau \propto$ $M_{\mathrm{ej}}^{2} E_{\mathrm{K}}^{-1}$, e.g., Maeda et al. 2003), the LC of model E03 declines slowly at late phase.

The characteristic parameters of the LC models are summarized in Table 5. In the lower energetic model, unburned $\mathrm{C}$ and $\mathrm{O}$ should be abundant, being $\sim 0.8 M_{\odot}$ in total. The presence of large amount of unburned $\mathrm{C}+\mathrm{O}$ is also seen in 3D deflagration model (Röpke et al. 2006), which is used in Phillips et al. (2007). Although our model is very simple, it mimics the properties of the $3 \mathrm{D}$ deflagration model. Our model is further verified by spectral modeling in $\S 6.3$.

10 The rise time of $15 \pm 1$ days is estimated by Phillips et al. (2007), using $3 \sigma$ upper limit at 15 days before B maximum. Our model LC assuming the rise time of 17 days is consistent with the upper limit. 


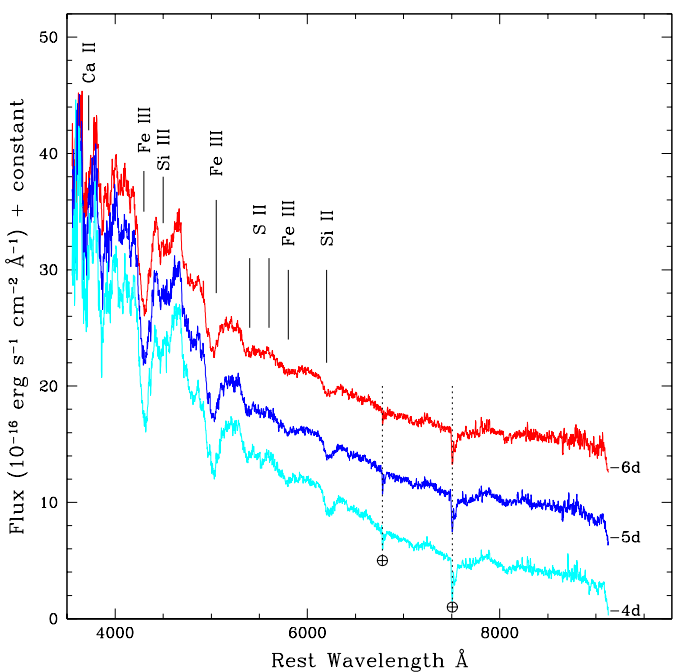

FIG. 8.- Pre-maximum spectral evolution of SN 2005hk. The phases marked are relative to date of $B$ maximum. The spectra have been corrected for the redshift of the host galaxy, but not corrected for the reddening. For clarity the spectra have been shifted vertically. The telluric lines have not been removed from the spectra and are marked with the symbol $\oplus$.

\section{THE SPECTRUM}

\subsection{Evolution during early phases}

The spectral evolution of SN 2005hk during the early phases are shown in Figure 8 - Figure 12. The premaximum spectra (ref. Fig.8) show a blue continuum, dominated by Fe III lines, with weak Si II and $\mathrm{Ca}$ II H\&K absorption (see §6.3.1). Comparing the pre-maximum spectrum with other normal Type Ia as well as peculiar Type Ia (Fig. 9), it is seen that the spectrum of SN $2005 \mathrm{hk}$ is very similar to that of SN 1991T. However, the minima of the $\mathrm{Ca}$ II H\& K, Fe II, Fe III and Si II lines, indicate that SN 2005hk has much lower expansion velocities that decrease from $\sim 6900 \mathrm{~km} \mathrm{~s}^{-1}$ on day -6 to $\sim 6200 \mathrm{~km} \mathrm{~s}^{-1}$ on day -4 . The similarity of the premaximum spectrum of SN 2005hk with SN 1991T-like events has also been noted by Phillips et al. (2007) \& Stanishev et al. (2006).

The post-maximum evolution of the spectrum of $\mathrm{SN}$ 2005hk is presented in Figures 10 and 12. The spectrum and its evolution are quite different from normal SNe Ia, as can be seen from Figure 11. The spectrum and its evolution closely matches that of SN 2002cx ( $\mathrm{Li}$ et al. 2003, Branch et al. 2004, Jha et al. 2006). The spectrum is dominated by Fe II and lines due to Co II, Na I and Ca II are also clearly present (see §6.3.1). Some Cr II lines may contribute to the absorption around $4800 \AA$ (Branch et al. 2004). The photospheric velocity decreases to $\sim 4000$ $\mathrm{km} \mathrm{s}^{-1}$ by day +10 . The flux in the blue continuum is found to decline steadily, and by day +14 , the continuum is quite weak below $4500 \AA$. This could also be an effect of line blanketing due to Fe II lines. The spectrum remains almost unchanged from day +10 to day +24 .

The spectrum of day +62 is very similar to that of day +45 , except for the narrowing of lines (ref. Fig.12). The lines in the $6500-6800 \AA$, identified with Co II (Branch et al. 2004), which by day +32 started weakening steadily with time (but they could be C II, see §6.3.1).

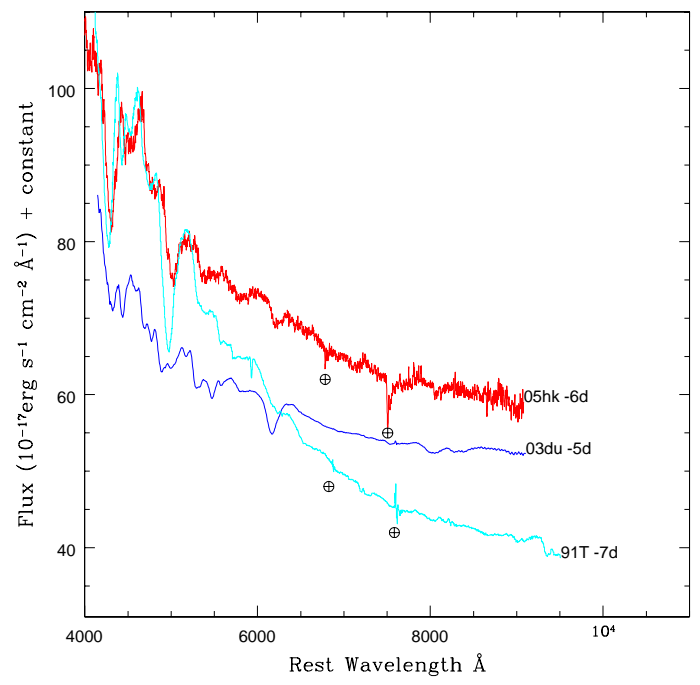

FIG. 9.- Pre-maximum spectrum of SN 2005hk compared with those of Type Ia SNe 1991T and 2003du at similar epoch. The phases marked are relative to date of $B$ maximum. The spectra have been corrected for the redshift of the host galaxy, but not corrected for the reddening. For clarity the spectra have been shifted vertically. The telluric lines have not been removed from the spectra of SNe 2005hk and 1991T, they are marked with the symbol $\oplus$.

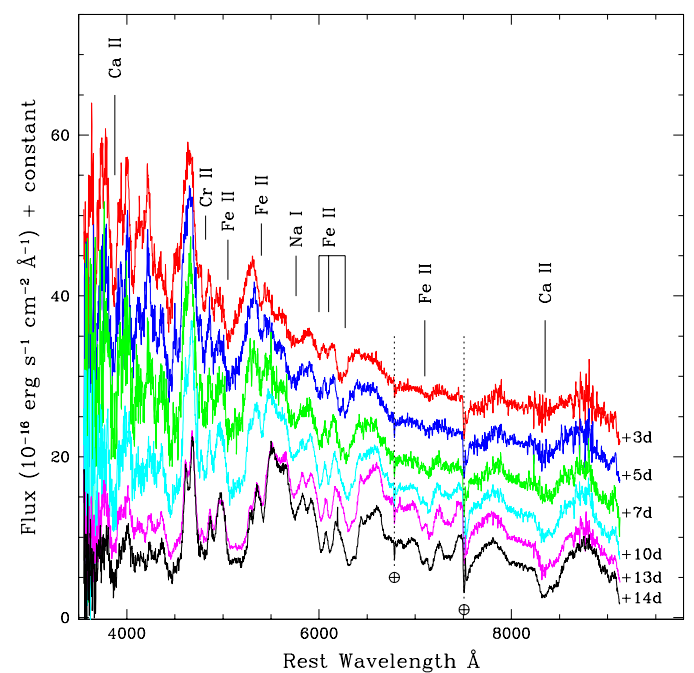

FIG. 10.- Spectral evolution of SN 2005hk from phase +3 days to +14 days relative to date of $B$ maximum. The spectra have been corrected for the redshift of the host galaxy, but not corrected for the reddening. For clarity the spectra have been shifted vertically. The telluric lines have not been removed from the spectra and are marked with the symbol $\oplus$. Line identification is based on Branch et al. (2004)

\subsection{The late phases}

The spectra of SN 2005hk were obtained during the late phase on days +228 and +377 (Fig. 13). As in the case of the early phase spectra, the spectrum of SN 2005hk during these phases is very different from the spectra of normal SNe Ia at similar phases. The spectrum of SN 2005hk is compared with those of other normal and peculiar Type Ia supernovae in Figure 14. The difference between the spectrum of SN 2005hk and spectra of other Type Ia supernovae is obvious. While the 


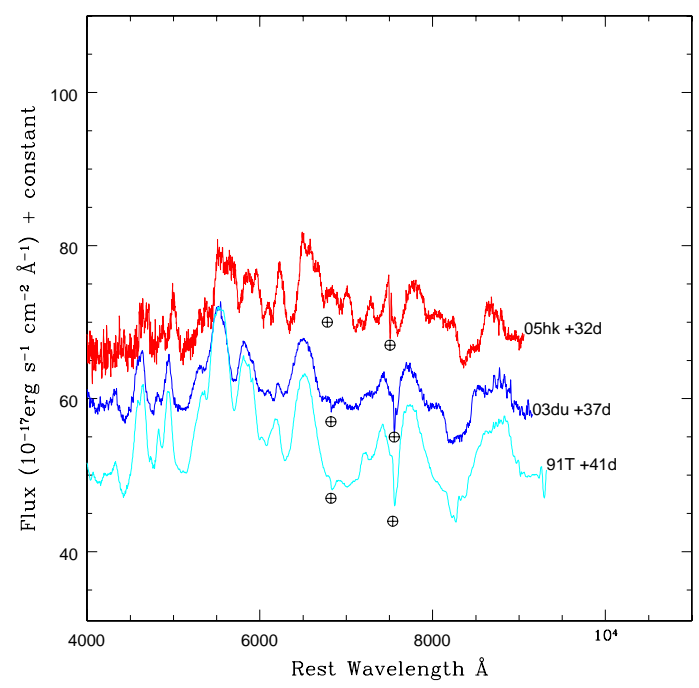

FIG. 11.- Post-maximum spectrum of SN 2005hk compared with those of Type Ia SNe 1991T and 2003du at similar epochs. The phases marked are relative to date of $B$ maximum. The spectra have been corrected for the redshift of the host galaxy, but not corrected for the reddening. For clarity the spectra have been shifted vertically. The telluric lines have not been removed from these spectra and are marked with the symbol $\oplus$.

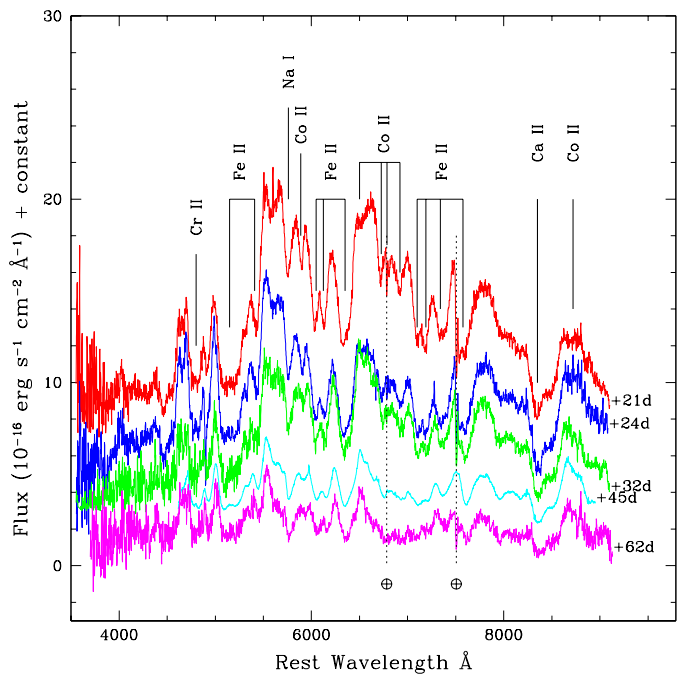

FIG. 12.- Spectral evolution of SN $2005 \mathrm{hk}$ from phase +21 days to +62 days relative to date of $B$ maximum. The spectra have been corrected for the redshift of the host galaxy, but not corrected for the reddening. For clarity the spectra have been shifted vertically. The telluric lines have not been removed from the spectra of SN 2005 hk taken with HCT and are marked with the symbol $\oplus$. The line identification is based on Branch et al. (2004).

other SNe Ia show blends of strong forbidden lines due to iron [Fe II] (5159 $\AA)$, [Fe III] (4500-4800 $\AA)$ and forbidden lines due to cobalt [Co III] (5890Å) (Kuchner et al. 1994) in their late phase spectrum, no signature of these lines is seen in the spectrum of SN 2005hk.

The late time spectrum of SN 2005hk is dominated by Fe II lines. The Ca II NIR lines are strong and Na I is also present. The spectra of days +228 and +377 (ref. Fig.13) also show forbidden lines due to [Ca II] 7291, $7234 \AA$ and $[\mathrm{Fe}$ II] at $7155 \AA$ and $7453 \AA$. The ratio of the fluxes of $\mathrm{Ca}$ II NIR and the [Ca II] lines on day +228

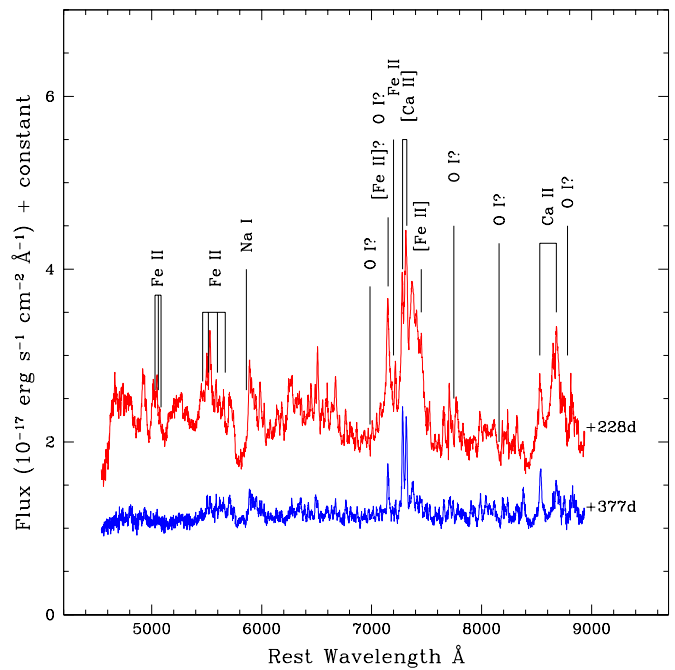

FIG. 13.- Late phase spectrum of SN 2005hk at +228 days and +377 days relative to date of $B$ maximum. The spectra have been corrected for the redshift of the host galaxy, but not corrected for the reddening. For clarity the spectra have been shifted vertically. The telluric lines have been removed from the spectrum. The line identification is based on Jha et al. (2006).

indicate densities of $\sim 10^{9} \mathrm{~cm}^{-3}$ in the line emitting region. By day +377 , the [Ca II] lines are stronger than the $\mathrm{Ca}$ II NIR lines, and the line ratios imply temperatures $\lesssim 4500 \mathrm{~K}$ and density $\sim 10^{8} \mathrm{~cm}^{-3}$ (Fransson \& Chevalier 1989). The mass ejected during the explosion can also be estimated using the number density arrived at with the Ca line ratios. Assuming that the ejecta is moving with a constant velocity of $1000 \mathrm{~km} \mathrm{~s}^{-1}$ over a period of $\sim$ 380 days after the explosion and a mean atomic weight $A=40$, the estimated mass of the ejecta is $\sim 0.25 M_{\odot}$. As pointed out by Jha et al. (2006) the mass of the ejecta arrived at is indicative as it does not take into account factors such as clumping or more complicated structures in the line emitting regions. It may however be noted that a high concentration of the mass in the central part is also suggested by the spectral modeling ( $\S 6.3$ ).

The shift in the central wavelength of the [Ca II] forbidden emission lines indicates an expansion velocity of $\sim 500 \mathrm{~km} \mathrm{~s}^{-1}$ on day +228 and $\sim 300 \mathrm{~km} \mathrm{~s}^{-1}$ on day +377 . The full width half maximum (FWHM) of the emission lines indicate a velocity dispersion of $\sim 1000$ $\mathrm{km} \mathrm{s}^{-1}$ on day +228 and $\sim 500 \mathrm{~km} \mathrm{~s}^{-1}$ on day +377 . Similarly, the emission component of Ca II NIR 8542 A line indicates an expansion velocity of $\sim 235 \mathrm{~km} \mathrm{~s}^{-1}$ on day +228 and $\sim 210 \mathrm{~km} \mathrm{~s}^{-1}$ on day +377 , with velocity dispersions $\sim 2100 \mathrm{~km} \mathrm{~s}^{-1}$ and $\sim 1200 \mathrm{~km} \mathrm{~s}^{-1}$, respectively. Low velocity O I at $7002 \AA$ and $7773 \AA$ absorption features are clearly identified in the late phase spectrum. These features were identified, for the first time in any SNe Ia, by Jha et al. (2006) in the "nebular" spectrum of SN 2002cx. The velocity of these lines, measured at the minimum is $\sim 800 \mathrm{~km} \mathrm{~s}^{-1}$. Though there is an overall similarity between the spectra of SN 2002cx and SN $2005 \mathrm{hk}$ at late phases, there are some noteworthy differences also. Comparing the spectrum of SN 2005hk on day +228 with the +227 day spectrum of SN $2002 \mathrm{cx}$ (Jha et al. 2006), it is found that the expansion velocities and the velocity dispersions are higher in SN 2005hk. The velocity dispersion based on the [Ca II] and the $\mathrm{Ca}$ II lines 


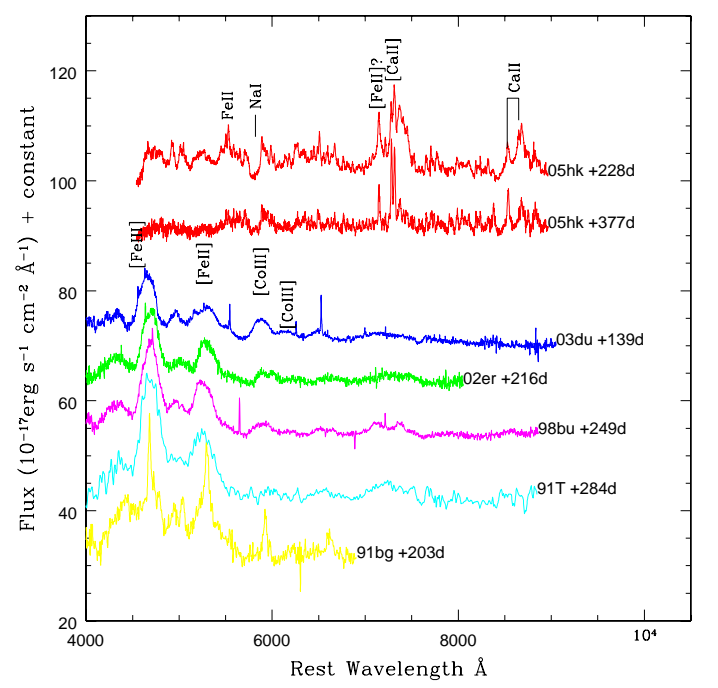

Fig. 14. - Late phase spectrum of SN $2005 \mathrm{hk}$ at +228 days and +377 days relative to date of $B$ maximum compared with those of other Type Ia SNe 2003du, 2002er, 1998bu, 1991T and 1991bg. The spectra have been corrected for the redshift of the host galaxy, but not corrected for the reddening. For clarity the spectra have been shifted vertically.

are in fact almost twice that estimated in SN 2002cx.

\subsection{Spectral modeling}

\subsubsection{Early Phases}

The observed spectra of SN 2005hk are further investigated by spectral modeling. For this purpose, we compute synthetic spectra using the Monte Carlo spectrum synthesis code developed by Mazzali \& Lucy (1993), Lucy (1999) and Mazzali (2000). We create model spectra at 5 epochs and compare them with the observed spectra $(-6,+3,+14,+24$ and +45 days from $B$ maximum). The rise time is assumed to be 17 days, as in the LC modeling ( 55.2 ).

The Monte Carlo spectrum synthesis code assumes a spherical, sharply defined photosphere in the ejecta as an inner boundary. By tracing rays of a number of photon packets emitted from the photosphere, the temperature structure in the SN atmosphere is computed through the flux at each radial point (see eq. (1) and (2) in Mazzali \& Lucy 1993). Radiative equilibrium is assumed for the temperature determination. For the interaction between photons and the SN ejecta, electron scattering and line scattering are taken into account. Sobolev approximation is used for line scattering. Sobolev optical depth is evaluated by computing ionization fractions and electron populations of each ion for a given temperature structure. Details of the physical assumptions adopted in the code are summarized in Mazzali \& Lucy (1993), Lucy (1999) and Mazzali (2000).

The input parameters of the code are the position (i.e., velocity, thanks to the homologous expansion) of the photosphere $\left(v_{\mathrm{ph}}\right)$ and the bolometric luminosity $(L)$. The parameter $L$ is the emergent luminosity. Since some photons emitted from the photosphere are back scattered into the photosphere, the luminosity at the photosphere (total photon luminosity emitted from the photosphere), is higher than the input bolometric luminosity $L$ (Mazzali \& Lucy 1993). As a density structure we use the less

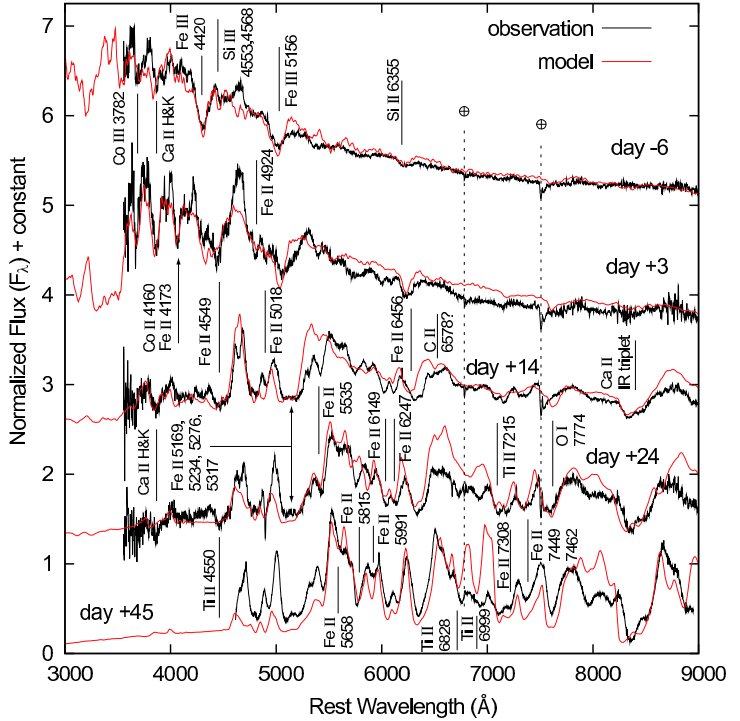

FIG. 15. - Comparison between the observed early phase spectra of SN 2005hk at $(-6,+3,+14,+24$ and +45 days from $B$ maximum, from top to bottom) and the synthetic spectra. Lines that make a major contribution to the absorptions are marked.

energetic model that gives the best agreement with the observed LC (model E03, see $\S 5$ and Table 5).

The mass fractions of elements are also required for the computation of the synthetic spectra. We treat the mass fractions as parameters rather than using the abundance distribution in the original LC model. The mass fractions of elements are assumed to be homogeneous above the photosphere. We determine the mass fraction of each element to give a good fit of the observed line depths. As time goes by, the photosphere recedes and the absorption lines are formed in the inner part of the ejecta. Nevertheless, we always assume a homogeneous abundance distribution at each epoch, although we change the mass fractions of the elements. The mass fractions derived by the modeling of each spectrum are thought to be a representative abundance ratio near the photosphere because the absorption lines are formed near the photosphere most efficiently.

The parameters in the best fitting cases are summarized in Table 6. Figure 15 shows the comparison between observed spectra and synthetic spectra. Synthetic spectra are reddened with $E(B-V)_{\text {Gal }}=0.022$ and $E(B-V)_{\text {Host }}=0.09$ (§4.). The distance modulus $\mu=33.46$ is assumed. In the figure, the flux and models at epoch are scaled and shifted for clarity.

The spectrum of SN $2005 \mathrm{hk}$ at day -6 is in good agreement with a synthetic spectrum computed with $\log L$ $\left(\mathrm{erg} \mathrm{s}^{-1}\right)=42.56$ and $v_{\mathrm{ph}}=6500 \mathrm{~km} \mathrm{~s}^{-1}$. The strong absorption features around $4300 \AA$ and $5000 \AA$ are mainly due to Fe III (Mazzali et al. 1995). The photospheric temperature is computed as $T=17000 \mathrm{~K}$ by taking into account the back scattering effect (Mazzali \& Lucy 1993). This high temperature makes the Fe III lines strong and also reproduces the color of the observed spectrum.

The temperature at the photosphere is relatively higher than that of normal Type Ia SNe at similar epoch, and it is comparable to the temperature at very early phase ( $\sim-10$ days; Tanaka et al. 2007). The weakness of Si II suggests that the outermost ejecta of SN 2005hk do not contain much $\mathrm{Si}(\mathrm{X}(\mathrm{Si}) \sim 0.02)$ as in normal $\mathrm{SNe}$ 
Ia $(\mathrm{X}(\mathrm{Si}) \gtrsim 0.5)$. The $\mathrm{S}$ I lines are similarly weak. The mass fraction of $\mathrm{S}$ is estimated as $\mathrm{X}(\mathrm{S}) \sim 0.02$. The $\mathrm{Ca}$ II lines are also very weak and there is no clear evidence of high velocity absorptions that is usually seen at pre-maximum epochs (Mazzali et al. 2005; Tanaka et al. 2006). No $\mathrm{C}$ lines are seen, which gives an upper limit of the $\mathrm{C}$ mass fraction $\mathrm{X}(\mathrm{C}) \lesssim 0.001$. The rest of the ejecta consist of oxygen, whose mass fraction is estimated as $\mathrm{X}(\mathrm{O}) \sim 0.86$.

The day +3 spectrum matches a synthetic spectrum with $\log (L)$ erg s ${ }^{-1}=42.66$ and $v_{\mathrm{ph}}=6000 \mathrm{~km} \mathrm{~s}^{-1}$. The temperature at the photosphere decreases $(T \sim 9,000 \mathrm{~K})$ from pre-maximum epochs. The low temperature allows some singly ionized ions such as Si II, Ca II and Fe II to appear in the spectrum.

The feature at $6300 \AA$ mainly consists of the Si II $\lambda 6355$, and it becomes stronger from pre-maximum epochs (Figs. 8 and 10). After the maximum, Fe lines begin to contribute to this feature (Figs.10 and 12). No $\mathrm{C}$ lines are visible, giving an upper limit of $\mathrm{C}$ mass fraction $\mathrm{X}(\mathrm{C}) \lesssim 0.001$. The mass fractions of $\mathrm{Si}, \mathrm{Ca}, \mathrm{Fe}$ and $\mathrm{Ni}$ can be estimated by the visible lines (Table 6). The mass fraction of $\mathrm{Si}$, stable $\mathrm{Fe}$ and ${ }^{56} \mathrm{Ni}$ required for the fitting of this spectrum are larger than those for premaximum spectra. Note again that we assume spatially homogeneous abundance distribution for each epoch.

After maximum brightness, the evolution of the spectral shape is not significant (Fig. 10). Almost all the absorption features are identified and they are mostly $\mathrm{O}$ I, $\mathrm{Ca}$ II, Fe II and Co II lines. The spectra are reproduced by almost the same element fractions (Table 6). Estimating the mass fraction of stable Fe separately with ${ }^{56} \mathrm{Ni}$ becomes difficult because more than $25 \%$ of ${ }^{56} \mathrm{Ni}$, which is one of the dominant elements, has decayed into ${ }^{56} \mathrm{Fe}$ at $\gtrsim 40$ days after the explosion.

An interesting feature is a weak absorption at $6400 \AA$ in the spectra at day +14 (and possibly in day +24 ). Branch et al. (2004) suggested that this is a Co II line. However, if we reproduce this feature by Co, the line blocking at $\lesssim 4000 \AA$ is too strong, making the flux there too low. This line could be reproduced by the $\mathrm{C}$ II $\lambda 6578$ line. This identification is, however, quite marginal. If we assume this feature is the $\mathrm{C}$ II, it requires $\mathrm{X}(\mathrm{C}) \sim$ 0.01. This is larger than the upper limit derived in the preceding spectra, which traces the abundances in the outer layer.

The $\mathrm{O}$ I $\lambda 7774$ line is always visible in the spectra from day +14 to +45 . The $\mathrm{O}$ I line is very weak in the earlier spectra, as a result of a severe suppression of $\mathrm{O}$ I fraction due to the high temperature. In fact, the modeling suggests a high $\mathrm{O}$ mass fraction in the outermost layers $(\mathrm{X}(\mathrm{O}) \gtrsim 0.75)$. These facts clearly indicate that unburned $\mathrm{O}$ exists in every place of the ejecta at $v>1000$ $\mathrm{km} \mathrm{s}^{-1}$. The mass of $\mathrm{O}$ derived from spectral modeling is discussed in $\S 6.3 .2$.

\subsubsection{Late phase}

The late phase spectra of SN 2005hk consist of a combination of P-Cygni profiles of permitted lines and emission features of forbidden lines (Fig.13). We note that no strong [O I] line is seen in the observed spectra. The presence of P-Cygni features suggests that the ejecta are not completely transparent even at such late epochs.
We study the late phase spectra using the model E03, which has similar properties to those of 3D deflagration model. Considering the observed spectra, there seems to be two major problems in this model.

(1) The ejecta of the E03 model become optically thin at the late phases. The photospheric velocity becomes zero at 250 days after the explosion if a constant line opacity $\left(0.1 \mathrm{~cm}^{2} \mathrm{~g}^{-1}\right)$ is assumed.

(2) In the E03 model, the mass of oxygen is as much as $0.44 M_{\odot}$. Such a model would give conspicuous emission lines of [O I] (e.g., Kozma et al. 2005).

First, we create synthetic spectra with the Monte Carlo code used in the modeling of early phase spectra. Here we assume the presence of the region emitting continuum light at the velocity of $v_{\mathrm{ph}}=250 \mathrm{~km} \mathrm{~s}^{-1}$. This could be realized if the central part of the ejecta is denser than that of the E03 model. A synthetic spectrum computed with $\log (L)\left(\mathrm{erg} \mathrm{s}^{-1}\right)=40.86$ and the element mass fraction same as at day +45 (blue line in Fig. 16) is in reasonable agreement with the observed spectrum at day +228 except for emission features at $7300 \AA$ ([Ca II $]$ ) and $8600 \AA$ (Ca II). The temperature at the photosphere is as low as $4400 \mathrm{~K}$. Most of the absorption lines are Fe II but some lines of neutral atoms such as $\mathrm{Na}$ I and Fe I are also found.

An absorption of the $\mathrm{O}$ I $\lambda 7774$ line is seen as discussed in $\S 6.2$ (also reported in SN 2002cx, Jha et al. 2006). In the synthetic spectrum, we could get the $\mathrm{O}$ I line with the mass fraction $\mathrm{X}(\mathrm{O}) \sim 0.5$. Therefore, we conclude that the unburned $\mathrm{O}$ is present in whole ejecta, down to very low velocities, suggesting a nearly completely mixed abundance distribution. This property is consistent with 3D deflagration models.

In modeling the early and late phase spectra, the mass fractions of elements are optimized assuming homogeneous abundance distribution above the photosphere at each epoch. If we assume these mass fractions are characteristic values at each velocity range of the ejecta, the mass of each element can be computed by integrating the density profile of the E03 model. In Table 5, the masses of $\mathrm{C}, \mathrm{O}, \mathrm{Si}$ and $\mathrm{Fe}$ group elements derived from spectral modeling are shown. Compared with the masses of the E03 model, the smaller amount of $\mathrm{C}$ and $\mathrm{Si}$, and larger amount of $\mathrm{O}$ are preferred by the spectra. The nuclear energy release corresponding to the element abundances obtained from the spectral fitting is $\sim 0.8 \times 10^{51} \mathrm{erg}$. Since the binding energy of the WD is $\sim 0.5 \times 10^{51} \mathrm{erg}$, this leads to a kinetic energy of $\sim 0.3 \times 10^{51} \mathrm{erg}$, which is consistent with the E03 model.

In the computation above, the cooling via lines is not considered, i.e., no emission lines would appear. In order to investigate the emission lines, we compute the late phase spectra using a nebular synthesis code and consider the combination with the synthetic photospheric spectrum (as in Mazzali et al. 2004).

The nebular code computes the deposition from $\gamma$-rays with a gray opacity $\left(0.025 \mathrm{~cm}^{2} \mathrm{~g}^{-1}\right)$ by Monte Carlo method. Positrons are assumed to be trapped in situ. To take into account the different channels in which the deposited energy is consumed, we assume $L_{\text {neb }}=f_{\text {neb }} L_{\gamma, e^{+}}$ where $L_{\gamma, e^{+}}$is the luminosity deposited by the $\gamma$-rays and positrons from $0.18 M_{\odot}$ of ${ }^{56} \mathrm{Co}$ (originally ${ }^{56} \mathrm{Ni}$ ). The fraction, $f_{\text {neb }}$, of $L_{\gamma, e^{+}}$is assumed to be balanced by cool- 


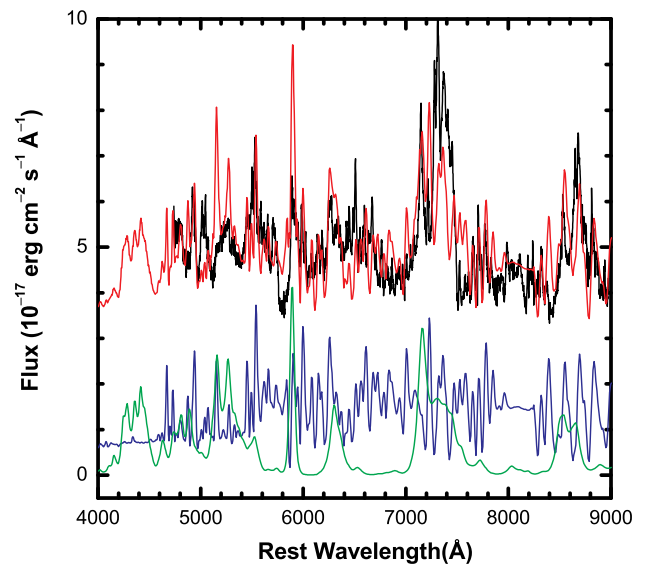

FIG. 16. - Spectrum of SN 2005hk at +228 days with respect to date of $B$ maximum (black line, $3 \times 10^{-17} \mathrm{erg} \mathrm{cm}^{-2} \mathrm{~s}^{-1} \AA^{-1}$ added to the flux for presentation), as compared to the synthetic "combined" spectrum (red line, the same amount added to the flux). The synthetic spectrum is a combination of the photospheric (blue line) and the nebular (green line) components.

ing via nebular emission lines, while the other fraction is by photospheric, allowed transitions as modeled by the early phase spectrum synthesis code. In other word, the luminosity $\left(1-f_{\text {neb }}\right) L_{\gamma, e^{+}}$is deposited below the photosphere, while the remaining luminosity $\left(f_{\text {neb }}\right) L_{\gamma, e^{+}}$is deposited into the optically thin layer above the photosphere. With this deposition luminosity (with varying $\left.f_{\text {neb }}\right)$, non-LTE rate equations are solved assuming the ionization-recombination equilibrium (Mazzali et al. 2001). Note that the code assumes that the ejecta are optically thin. For the calculation, we assume homogeneous distribution of elements and ${ }^{56} \mathrm{Co}$. The masses of these elements are set to be consistent with the E03 model, i.e., $M\left({ }^{56} \mathrm{Co}\right)=0.18 M_{\odot}$ and $M(\mathrm{O})=0.4 M_{\odot}$.

Figure 16 shows the combination of the photospheric (as described in the first part of this section, with the luminosity corresponding to $\left.M\left({ }^{56} \mathrm{Co}\right)=0.27 M_{\odot}{ }^{11}\right)$ and nebular components with $f_{\text {neb }}=0.5$. We find that the $[\mathrm{O} \mathrm{I}] \lambda \lambda 6300,6363$ doublet is not strong in the model spectra. Indeed, the resulting $\left[\begin{array}{ll}\mathrm{O} & \mathrm{I}\end{array}\right]$ is so weak that it does not contradict with the non-detection of the $[\mathrm{O} \mathrm{I}]$, if $f_{\text {neb }} \lesssim 0.5$, as is consistent with the observed, allowed line-dominated spectrum.

This is an outcome of (a) the high density of the ejecta and of (b) the assumption that the elements, especially $\mathrm{O}$ and $\mathrm{Fe}$, are homogeneously, thus microscopically, mixed together with one another. With these two conditions, the deposited luminosity is effectively consumed by copious $[\mathrm{Fe}$ II $]$ lines rather than $[\mathrm{O} \mathrm{I}]{ }^{12}$. The high density is a direct consequence of the low energy SN Ia model. However, the complete mixing is a rather extreme assumption, so that we need more detailed modeling of the late phase spectra of SN 2005hk.

With the nebular component, we obtain better fits

\footnotetext{
11 This luminosity is higher than that of the E03 model because the late spectrum taken with Subaru is calibrated against the photometry that is brighter than the model LC (Fig. 6) at 250 days after the explosion.

12 Indeed, [Fe II] around $5200 \AA$ is too strong in the model spectrum, thus smaller value of $f_{\text {neb }}$ is favored.
}

compared to purely photospheric component, in the following aspects: (i) Strong emission features below $\sim 5500 \AA$, (ii) $[\mathrm{Fe}$ II $] \lambda 7155$ and $[\mathrm{Fe}$ II $] \lambda 7172$, (iii) $[\mathrm{Ca}$ II $] \lambda \lambda 7291,7324$, and (iv) Ca II IR and [C I] $\lambda 8727$.

The synthetic nebular spectrum for the E03 model results in broader lines than the observed nebular emissions. Typical example is [Ca II] $\lambda \lambda 7291,7324$. The two components are separated in the observations, but blended in the model. This indicates that the nebular line emitting region is indeed more centrally concentrated than the model. This may again suggest the central part being denser than the E03 model.

In summary, the major problems (1) and (2) above could be solved if the ejecta are denser than in the original model and the elements are microscopically mixed. Then, the ejecta at late phases consist of three parts: the optically thick innermost part $\left(v \lesssim 250 \mathrm{~km} \mathrm{~s}^{-1}\right)$, the optically thin region emitting forbidden lines $(v \lesssim 700$ $\mathrm{km} \mathrm{s}^{-1}$, i.e., the width of the [Ca II] lines), and the outer layer which is thin even to $\gamma$-rays.

\section{DISCUSSION}

We have studied the bolometric LC and early and late phase spectra by computing synthetic LC and spectra. We construct a simple, less energetic model by scaling the kinetic energy of W7 down to $0.3 \times 10^{51} \mathrm{erg}$. This model (E03) explains the LC of SN 2005hk from early to late phase. The ejecta of the model contain as much as $\sim 0.8 M_{\odot}$ of unburned $\mathrm{C}+\mathrm{O}$ material. In this sense, our less energetic model is similar to the 3D deflagration model.

Branch et al. (2004), Jha et al. (2006) and Phillips et al. (2007) suggested that the properties of SN 2002cxlike objects are similar to those of the 3D deflagration model. We confirm this suggestion by modeling the LC until late phase for the first time. We also find that the early phase spectra of SN 2005hk are also explained by lower energetic model. The O I absorption is always seen until late phase, suggesting that the elements in the ejecta are mixed almost homogeneously. This is also consistent with the 3D deflagration model.

The late phase spectra of SN 2005hk do not show strong [O I] line and do show P-cygni profiles of permitted lines. They seem inconsistent with what $3 \mathrm{D}$ deflagration model predicts, at a first look. To study this in detail, we modeled the late phase spectra of SN $2005 \mathrm{hk}$ by a combination of the photospheric and nebular components. We find that the innermost region of the ejecta should be denser and more centrally concentrated than that of our model in order to make an optically thick region and a smaller emitting region of nebular lines. The absence of the [O I] line is explained if the elements are microscopically mixed.

In summary, our less energetic explosion model or 3D deflagration model seems to be compatible with the late phase spectrum of SN 2005hk (and of SN 2002cx-like objects) (1) if the density of the central region is higher than that of our scaled model and (2) if the elements are microscopically mixed, although it is a question how such extreme conditions are realized.

The nature of this class of objects can be studied further if observations become available at phases later than presented here. SN 2005hk is still not completely trans- 
parent at $\sim 400$ days after the explosion. Only when the ejecta become completely thin, useful information on the structure of the innermost ejecta can be obtained.

If there is a sub-class of SNe Ia with a lower kinetic energy than in normal SNe Ia, it could be harmful for cosmological use of SNe Ia. To show the effect, we construct a model less energetic than model E03 by scaling the energy of W7 model to $0.08 \times 10^{51} \mathrm{erg}(\sim 1 / 4$ of the kinetic energy of the E03 model). If the deflagration is very weak and slow, such a situation will be realized. Since the nuclear energy release is only slightly higher than the binding energy of the WD, such an explosion could be followed after a pulsation (pulsating deflagration model, e.g., Nomoto, Sugimoto \& Neo 1976). Since the mass of ${ }^{56} \mathrm{Ni}$ synthesized by such an explosion is thought to be smaller than that of the E03 model, we set $M\left({ }^{56} \mathrm{Ni}\right)=0.09 M_{\odot}(1 / 2$ of E03). We call this model E008, and the parameters are summarized in Table 5.

In Figure 6, the synthetic LC with the E008 model is shown as a magenta line. The LC has a fainter peak magnitude than that of the E03 model because of the smaller amount of ${ }^{56} \mathrm{Ni}$. However, the decline rate of the LC after maximum is slower than in the E03 model because of the lower kinetic energy of the ejecta. This trend is opposite to the well-known LC-width relation in $\mathrm{SNe} I a$, i.e., brighter SNe Ia declines more slowly.

Such explosions can be distinguished by the late phase LC. Since less energy leads to an efficient trapping of $\gamma$-rays at late phases, the decline rate of the LC at $\gtrsim$ 100 days after the explosion is slower. This is clearly seen in the synthetic LC (Figure 6). Although the peak magnitude of the E008 model is fainter than that of the E03 by $\sim 1 \mathrm{mag}$, the late phase luminosities of these two models are comparable.

A recently discovered supernova, SN 2007qd may belong to this sub-class (SN 2002cx-like; Bassett et al. 2007, Goobar et al. 2007). This supernova was reported to have reached a fainter maximum than SN 2002cx and SN 2005hk and lower line velocity, being roughly half that of 2002cx or 2005hk. Such properties are realized if the kinetic energy of the ejecta is about quarter that of SN 2002cx (similar to the E008 model), because of the relation of $E_{\mathrm{K}} \propto v^{2}$. If this is true, the LC of SN 2007qd will evolve very slowly as shown in the magenta line in Figure 6.

\section{CONCLUSIONS}

Photometric and spectrosopic data on the peculiar SN 2005hk are presented. The $B$ band light curve of SN 2005hk shows comparable pre-maximum brightening, faster decline in the initial $\sim 20$ days past maximum and slower decline beyond $\sim 50$ days past maximum, as compared to normal Type Ia supernovae. The fainter peak bolometric luminosity indicates synthesis of small amount of ${ }^{56} \mathrm{Ni}$ in the explosion. Low expansion velocity of the ejecta together with fainter peak luminosity is explained by an explosion with lower kinetic energy. A reasonable fit to the bolometric light curve of SN $2005 \mathrm{hk}$ is achieved with a less energetic $\left(0.30 \times 10^{51} \mathrm{erg}\right)$ model which synthesized $0.18 M_{\odot}$ of ${ }^{56} \mathrm{Ni}$. The light curve evolution is similar to SN 2002cx.

The pre-maximum spectrum of SN 2005hk is similar to that of SN 1991T, with much lower expansion velocity. The spectral evolution of SN 2005hk is very similar to SN 2002cx. The spectrum of SN 2005hk at late phases (> 200 days) is similar to SN 2002cx, except for higher expansion velocities and higher velocity dispersions. The presence of P-Cygni profiles in the late phase spectrum of SN 2005hk indicates that the ejecta have not become optically thin till our last observation. Modeling of the pre-maximum spectra of SN 2005hk indicates a relatively higher temperature which makes Fe III lines strong. The presence of weak O I line at $\lambda 7774$ at almost all epochs is modeled as a consequence of high abundance of completely mixed unburned oxygen in the ejecta. The late phase spectra of SN 2005hk are modeled as a combination of the photospheric and nebular components, with the nebular line emitting region being more centrally concentrated than is expected in the case of a lower energetic model.

We are thankful to the anonymous referee for valuable comments. This work has been carried out under the INSA (Indian National Science Academy) JSPS (Japan Society for Promotion of Science) exchange programme. This research was supported in part by the National Science Foundation under Grant No. PHY05-51164 and grant-in-Aid for Scientific Research (18104003, 18540231) and the 21st Century COE Program (QUEST) from the JSPS and MEXT of Japan. M.T. and N.T. are supported through the JSPS Research Fellowships for Young Scientists. K.M. is supported through the JSPS Postdoctoral Fellowships for Research Abroad. This work has used data collected at Subaru Telescope, which is operated by the National Astronomical Observatory of Japan. We thank J. Deng for useful discussions and S. Srividya for help in observations and data reduction. We thank all the observers of the 2-m HCT (operated by the Indian Institute of Astrophysics), who kindly provided part of their observing time for the supernova observations. This work has made use of the NASA Astrophysics Data System and the NASA/IPAC Extragalactic Database (NED) which is operated by Jet Propulsion Laboratory, California Institute of Technology, under contract with the National Aeronautics and Space Administration.

\section{REFERENCES}

Altavilla, G. et al., 2004, MNRAS, 349, 1344

Anupama, G. C., Sahu, D. K., Jose, J. 2005, A\&A, 429, 667

Arnett, W.D., 1982, ApJ, 253, 785

Barentine et al., 2005, CBET 268.

Bassett, B. et al. 2007, CBET 1137.

Bessell, M.S., Castelli, F. \& Plez, B., 1998, A\&A, 333, 231

Blinnikov, S.I., et al. 2006, A\&A, 453, 229

Branch, D., Baron, E., Thomas, R. C., Kasen, D., Li, W., Filippenko, A. V. 2004, PASP, 116, 903
Branch, D., Fisher, A. \& Nugent, P., AJ, 1993, 106, 2383

Burket \& Li 2005, IAUC, 8625

Cardelli, J.A., Clayton, G.C., Mathis, J.S., 1989, ApJ, 345, 245

Chornock, R., Filippenko, A. V., Branch, D., Foley, R. J., Jha, S., Li, W. 2006, PASP, 118, 722

Conley, A., et al. 2006, AJ, 132, 1707

Contardo, G., Leibundgut, B., Vacca, W.D., 2000, A\&A, 359, 876 
TABLE 1

MAGNitudes FOR THE SEQUENCE OF SECONDARY STANDARD STARS IN THE FIELD OF SN 2005 HK.

\begin{tabular}{lccccc}
\hline \hline $\mathrm{ID}$ & $\mathrm{U}$ & $\mathrm{B}$ & $\mathrm{V}$ & $\mathrm{R}$ & $\mathrm{I}$ \\
\hline 1 & $15.896 \pm 0.020$ & $15.425 \pm 0.006$ & $14.643 \pm 0.004$ & $14.174 \pm 0.012$ & $13.777 \pm 0.005$ \\
2 & $17.257 \pm 0.025$ & $16.859 \pm 0.007$ & $16.067 \pm 0.005$ & $15.579 \pm 0.010$ & $15.149 \pm 0.010$ \\
3 & $19.175 \pm 0.035$ & $17.696 \pm 0.002$ & $16.375 \pm 0.009$ & $15.564 \pm 0.007$ & $14.886 \pm 0.010$ \\
4 & $16.824 \pm 0.025$ & $16.954 \pm 0.010$ & $16.449 \pm 0.016$ & $16.097 \pm 0.016$ & $15.750 \pm 0.009$ \\
5 & $17.842 \pm 0.027$ & $17.645 \pm 0.010$ & $16.902 \pm 0.009$ & $16.440 \pm 0.018$ & $16.003 \pm 0.008$ \\
6 & $17.094 \pm 0.022$ & $16.870 \pm 0.016$ & $16.150 \pm 0.010$ & $15.706 \pm 0.017$ & $15.275 \pm 0.005$ \\
7 & $17.834 \pm 0.032$ & $17.213 \pm 0.011$ & $16.210 \pm 0.012$ & $15.646 \pm 0.022$ & $15.108 \pm 0.004$ \\
8 & $19.134 \pm 0.040$ & $18.194 \pm 0.020$ & $17.175 \pm 0.014$ & $16.571 \pm 0.013$ & $16.055 \pm 0.004$ \\
9 & $16.366 \pm 0.024$ & $16.470 \pm 0.007$ & $16.115 \pm 0.007$ & $15.871 \pm 0.012$ & $15.623 \pm 0.002$ \\
10 & $19.516 \pm 0.042$ & $18.162 \pm 0.020$ & $16.944 \pm 0.010$ & $16.201 \pm 0.021$ & $15.553 \pm 0.001$ \\
11 & & $18.059 \pm 0.008$ & & $16.782 \pm 0.016$ & \\
& & & & & \\
\hline
\end{tabular}

${ }^{*}$ The stars are identified in Figure. 1

Filippenko, A. V., Richmond, M. W., Branch, D., Gaskell, M., Herbst, W., Ford, C. H., Treffers, R.R., Matheson, T., Ho, L. C. et al. 1992, AJ, 104, 1543

Fransson, C. \& Chevalier, R.A., 1989, ApJ, 343, 323

Goldhaber, G., et al. 2001, ApJ, 558, 359

Goobar, et al., 2007, CBET, 1137, 2

Hamuy, M., Phillips, M.M., Suntzeff, N.B., Schommer, R.A., Maza, J., Avils, R. 1996a, AJ, 112, 2391

Hamuy, M., Phillips, M.M., Suntzeff, N.B., Schommer, R.A., Maza, J., Smith, R.C., Lira, P., Avils, R. 1996b, AJ, 112, 2438

Iwamoto, K. 1997, PhD thsis, University of Tokyo

Iwamoto, K., et al. 2000, ApJ, 534, 660

Jha, S., Branch, D., Chornock, R., Foley, R. J., Li, W., Swift, B. J., Casebeer, D., Filippenko, A. V., AJ, 2006, 132, 189

Kashikawa, N., Aoki, K., Asai, R., Ebizuka, N., Inata, M., Iye, M., Kawabata, K. S., Kosugi, G., Ohyama, Y., Okita, K., 2002, PASJ, 54, 819

Kozma, C., et al. 2005, A\&A, 437, 983

Kuchner, M.J., Kirshner, R.P., Pinto, P.A., Leibundgut, B., 1994, 426, L89

Landolt, A.U., 1992, AJ, 104, 340

Li, W., Filippenko, A. V.; Treffers, R. R.; Riess, A. G.; Hu, J.; Qiu, Y., ApJ, 2001, 546, 734

Li, W., Filippenko, A. V., Chornock, R., Berger, E., Berlind, P., Calkins, M. L., Challis, P., Fassnacht, C. Jha, S., Kirshner, R.P. PASP, 2003, 115, 453

Lira, P. 1996, Master's thesis, Univ. Chile

Lira, P., Hamuy, M., Wells, L.A., et al. 1998, AJ, 115, 234

Lucy, L.B. 1999, A\&A, 345, 211

Maeda, K., Mazzali, P. A., Deng, J., Nomoto, K., Yoshii, Y., Tomita, H., \& Kobayashi, Y. 2003, ApJ, 593, 931

Mazzali, P.A. 2000, A\&A, 363, 705

Mazzali, P.A. \& Lucy, L.B. 1993, A\&A, 279, 447

Mazzali, P. A., Danziger, I. J., Turatto, M. 1995, A\&A, 297, 509
Mazzali, P.A., Nomoto, K., Maeda, K., \& Patat, F, 2001, ApJ, 559, 1047

Mazzali, P. A., Deng, J., Maeda, K., Nomoto, K., Filippenko, A.

V., \& Matheson, T. 2004, ApJ, 614, 858

Mazzali, P.A., et al. 2005, ApJ, 623L, 37

Nomoto, K., Sugimoto, D., \& Neo, S. 1976, Ap\&SS, 39, L37

Nomoto, K., Thielemann, F.-K., \& Yokoi, K. 1984, ApJ, 286, 644

Phillips, M.M, Wells, L.A., Suntzeff, N.B., et al. 1992, AJ, 103, 1632

Phillips, M.M., Lira, P., Suntzeff, N.B., Schommer, R.A., Hamuy, M., Jose, M. 1999, AJ, 118, 1766

Phillips, M. M., Li, W., Frieman, J. A., Blinnikov, S. I., DePoy, D., Prieto, J. L., Milne, P., Contreras, C., Folatelli, G.; Morrell, N. et al. 2007, PASP, 119, 360

Richmond, M.W., Treffers, R.R., Filippenko, A.V. et al, 1995, AJ, 109,2121

Röpke, F.K., Gieseler, M., Reinecke, M., Travaglio, C., \& Hillebrandt., W. 2006, A\&A, 453, 203

Schelgel, D.J., Finkbeiner, D.P., Davis, M., 1998, ApJ, 500, 525

Stanishev, V., Taubenberger, S., Blanc, G., Anupama, G. C., Benetti, S., Cappellaro, E., Elias-Rosa, N., Feron, C., Goobar, A., Krisciunas, K. et al. 2006, astroph/0611354

Suntzeff, N.B.: 1996, in R. McCray and Z. Wang (eds.), Supernovae and Supernova Remnants, p. 41, Cambridge Univ. Press, Cambridge

Tanaka, M., Mazzali, P. A., Maeda, K., \& Nomoto, K. 2006, ApJ, 645,470

Tanaka, M., et al. 2007, ApJ, in press (arxiv:0712.2823)

Turatto, M., Benetti, S., Cappellaro, E., Danziger, I. J., della Valle, M., Gouiffes, C., Mazzali, P. A., Patat, F. 1996, MNRAS, 283, 1 . 
TABLE 2

Photometric OBSERVATions OF SN 2005hK

\begin{tabular}{|c|c|c|c|c|c|c|c|}
\hline Date & $\begin{array}{c}\text { J.D. } \\
2453000+\end{array}$ & $\begin{array}{c}\text { Phase* } \\
\text { (days) }\end{array}$ & $\mathrm{U}$ & B & $\mathrm{V}$ & $\mathrm{R}$ & I \\
\hline $05 / 11 / 2005$ & 680.06 & -5.22 & & $16.126 \pm 0.014$ & $16.167 \pm 0.018$ & $16.023 \pm 0.014$ & $16.060 \pm 0.032$ \\
\hline $06 / 11 / 2005$ & 681.04 & -4.25 & $15.482 \pm 0.046$ & $16.066 \pm 0.012$ & $16.047 \pm 0.011$ & $15.934 \pm 0.021$ & $15.979 \pm 0.016$ \\
\hline $07 / 11 / 2005$ & 682.03 & -3.27 & $15.181 \pm 0.086$ & $16.004 \pm 0.046$ & $16.007 \pm 0.027$ & $15.862 \pm 0.016$ & $15.866 \pm 0.039$ \\
\hline $08 / 11 / 2005$ & 683.06 & -2.25 & $15.515 \pm 0.060$ & $15.966 \pm 0.018$ & $15.900 \pm 0.018$ & $15.784 \pm 0.013$ & $15.798 \pm 0.024$ \\
\hline $13 / 11 / 2005$ & 688.04 & 2.66 & $15.741 \pm 0.119$ & $16.022 \pm 0.069$ & $15.739 \pm 0.050$ & $15.631 \pm 0.034$ & $15.577 \pm 0.051$ \\
\hline $15 / 11 / 2005$ & 690.13 & 4.73 & $15.956 \pm 0.066$ & $16.208 \pm 0.027$ & $15.710 \pm 0.024$ & $15.564 \pm 0.017$ & $15.523 \pm 0.016$ \\
\hline $16 / 11 / 2005$ & 691.20 & 5.79 & $16.030 \pm 0.058$ & $16.260 \pm 0.021$ & $15.763 \pm 0.023$ & $15.563 \pm 0.016$ & $15.416 \pm 0.018$ \\
\hline $17 / 11 / 2005$ & 692.14 & 6.72 & $16.222 \pm 0.062$ & $16.413 \pm 0.020$ & $15.779 \pm 0.020$ & $15.568 \pm 0.019$ & $15.466 \pm 0.027$ \\
\hline $19 / 11 / 2005$ & 694.13 & 8.69 & $16.572 \pm 0.116$ & $16.671 \pm 0.021$ & $15.840 \pm 0.013$ & $15.553 \pm 0.011$ & $15.427 \pm 0.025$ \\
\hline $22 / 11 / 2005$ & 697.06 & 11.58 & $17.212 \pm 0.037$ & $17.132 \pm 0.012$ & $16.019 \pm 0.008$ & $15.649 \pm 0.015$ & $15.466 \pm 0.015$ \\
\hline $01 / 12 / 2005$ & 706.06 & 20.48 & $18.620 \pm 0.021$ & $18.260 \pm 0.021$ & $16.704 \pm 0.009$ & $16.126 \pm 0.015$ & $15.796 \pm 0.019$ \\
\hline $05 / 12 / 2005$ & 710.03 & 24.40 & & $18.494 \pm 0.011$ & $16.925 \pm 0.011$ & $16.364 \pm 0.013$ & $15.970 \pm 0.019$ \\
\hline $08 / 12 / 2005$ & 713.02 & 27.36 & & $18.600 \pm 0.019$ & $17.076 \pm 0.010$ & $16.501 \pm 0.017$ & $16.106 \pm 0.009$ \\
\hline $12 / 12 / 2005$ & 717.03 & 31.32 & & $18.758 \pm 0.033$ & $17.237 \pm 0.024$ & $16.646 \pm 0.025$ & $16.251 \pm 0.018$ \\
\hline $17 / 12 / 2005$ & 722.06 & 36.29 & & $18.832 \pm 0.016$ & $17.362 \pm 0.012$ & $16.826 \pm 0.013$ & \\
\hline $23 / 12 / 2005$ & 728.13 & 42.29 & & $18.846 \pm 0.096$ & $17.607 \pm 0.051$ & $17.053 \pm 0.041$ & $16.593 \pm 0.044$ \\
\hline $26 / 12 / 2005$ & 730.79 & 44.92 & & $18.859 \pm 0.010$ & & $17.150 \pm 0.017$ & \\
\hline $26 / 12 / 2005$ & 731.14 & 45.27 & & $18.944 \pm 0.024$ & $17.605 \pm 0.010$ & $17.101 \pm 0.009$ & $16.698 \pm 0.023$ \\
\hline $28 / 12 / 2005$ & 733.07 & 47.17 & & $19.017 \pm 0.014$ & $17.618 \pm 0.013$ & $17.158 \pm 0.008$ & $16.749 \pm 0.008$ \\
\hline $12 / 01 / 2006$ & 748.06 & 61.99 & & $19.204 \pm 0.031$ & $17.920 \pm 0.014$ & $17.501 \pm 0.011$ & $17.142 \pm 0.027$ \\
\hline $14 / 01 / 2006$ & 750.05 & 63.96 & & $19.325 \pm 0.045$ & $18.038 \pm 0.035$ & $17.548 \pm 0.027$ & $17.203 \pm 0.015$ \\
\hline $30 / 06 / 2006$ & 917.04 & 230.03 & & $21.578 \pm 0.033$ & & $20.140 \pm 0.022$ & \\
\hline $04 / 07 / 2006$ & 921.40 & 233.31 & & & $20.940 \pm 0.044$ & & \\
\hline $18 / 07 / 2006$ & 935.40 & 247.14 & & & & $20.474 \pm 0.036$ & \\
\hline $20 / 09 / 2006$ & 999.39 & 310.38 & & & $22.733 \pm 0.168$ & $21.633 \pm 0.099$ & \\
\hline $27 / 11 / 2006$ & 1066.73 & 376.98 & & $24.206 \pm 0.124$ & & $21.776 \pm 0.036$ & \\
\hline
\end{tabular}

* Observed phase with respect to the epoch of maximum in $B$ band (JD 2453685.34), corrected for the (1+z) timedilation factor using the host galaxy redshift $\mathrm{z}=0.0118$

TABLE 3

LOG OF SPECTROSCOPIC OBSERVATIONS OF SN 2005HK

\begin{tabular}{lcrrl}
\hline \hline Date & $\begin{array}{c}\text { J.D. } \\
2453000+\end{array}$ & $\begin{array}{c}\text { Phase* } \\
\text { (days) }\end{array}$ & $\begin{array}{c}\text { Range } \\
\AA\end{array}$ & Telescope \\
\hline $04 / 11 / 05$ & 679.10 & -6.17 & $3500-7000 ; 5200-9100$ & HCT \\
$05 / 11 / 05$ & 680.06 & -5.22 & $3500-7000 ; 5200-9100$ & HCT \\
$06 / 11 / 05$ & 681.06 & -4.23 & $3500-7000 ; 5200-9100$ & HCT \\
$13 / 11 / 05$ & 688.06 & 2.69 & $3500-7000 ; 5200-9100$ & HCT \\
$15 / 11 / 05$ & 690.25 & 4.85 & $3500-7000 ; 5200-9100$ & HCT \\
$17 / 11 / 05$ & 692.10 & 6.68 & $3500-7000 ; 5200-9100$ & HCT \\
$20 / 11 / 05$ & 695.27 & 9.91 & $3500-7000 ; 5200-9100$ & HCT \\
$23 / 11 / 05$ & 698.19 & 12.70 & $3500-7000 ; 5200-9100$ & HCT \\
$24 / 11 / 05$ & 699.18 & 13.68 & $3500-7000 ; 5200-9100$ & HCT \\
$01 / 12 / 05$ & 706.09 & 20.50 & $3500-7000 ; 5200-9100$ & HCT \\
$05 / 12 / 05$ & 710.08 & 24.45 & $3500-7000 ; 5200-9100$ & HCT \\
$13 / 12 / 05$ & 718.02 & 32.30 & $3500-7000 ; 5200-9100$ & HCT \\
$26 / 12 / 05$ & 730.81 & 44.94 & $4000-9000$ & Subaru \\
$12 / 01 / 06$ & 748.09 & 62.02 & $3500-7000 ; 5200-9100$ & HCT \\
$30 / 06 / 06$ & 916.5 & 228.46 & $4700-9000$ & Subaru \\
$27 / 11 / 06$ & 1067.05 & 377.26 & $4700-9000$ & Subaru \\
& & & & \\
\hline
\end{tabular}

* Observed phase with respect to the epoch of maximum in $B$ band (JD 2453685.34), corrected for the (1+z) time-dilation factor using the host galaxy redshift $\mathrm{z}=0.0118$ 
TABLE 4

Photometric PARAmeters FOR SN 2005hK

\begin{tabular}{|c|c|c|c|c|}
\hline Data & $B$ & $V$ & $R$ & $I$ \\
\hline $\begin{array}{l}\text { epoch of } \max ^{*} \\
\text { magnitude at } \max \\
\Delta m_{15}(B)\end{array}$ & $\begin{array}{r}685.34 \pm 0.4 \\
15.91 \pm 0.03 \\
1.68 \pm 0.05\end{array}$ & $\begin{array}{l}689.49 \pm 0.8 \\
15.71 \pm 0.04\end{array}$ & $\begin{array}{l}691.78 \pm 0.2 \\
15.55 \pm 0.02\end{array}$ & $\begin{array}{l}693.93 \pm 0.3 \\
15.43 \pm 0.03\end{array}$ \\
\hline colors at $\mathrm{B} \max ^{* *}$ & & $\begin{array}{r}B-V \\
-0.03 \pm 0.04\end{array}$ & $\begin{array}{r}V-R \\
0.08 \pm 0.03\end{array}$ & $\begin{array}{r}R-I \\
-0.02 \pm 0.06\end{array}$ \\
\hline $\begin{array}{l}\text { decline rate days } 20-45\left(\operatorname{mag} \text { day }^{-1}\right) \\
\text { decline rate days } 230-380\left(\operatorname{mag}^{-1}\right)\end{array}$ & 0.021 & $\begin{array}{l}0.027 \\
0.015\end{array}$ & $\begin{array}{l}0.031 \\
0.011\end{array}$ & 0.031 \\
\hline
\end{tabular}

${ }^{*}$ JD $2453000+$

${ }^{* *}$ colors are corrected for reddening $E(B-V)_{\text {total }}=0.11$

TABLE 5

Characteristic Quantities of the Models

\begin{tabular}{lcccccc}
\hline \hline Model & $E_{\mathrm{K}}{ }^{\mathrm{a}}$ & $M\left({ }^{56} \mathrm{Ni}\right)^{\mathrm{b}}$ & $M(\mathrm{C})^{\mathrm{c}}$ & $M(\mathrm{O})^{\mathrm{d}}$ & $M(\mathrm{Si})^{\mathrm{e}}$ & $M(\text { Fe group })^{\mathrm{f}}$ \\
\hline W7 (92A) & 1.2 & 0.17 & 0.038 & 0.12 & 0.16 & 0.95 \\
W7 (91bg) & 1.2 & 0.088 & 0.038 & 0.12 & 0.16 & 0.95 \\
E03 & 0.30 & 0.18 & 0.36 & 0.44 & 0.074 & 0.45 \\
E008 & 0.08 & 0.09 & 0.44 & 0.53 & 0.053 & 0.32 \\
\hline Spectra & - & - & $<0.011$ & 0.84 & 0.023 & 0.44 \\
\hline
\end{tabular}

${ }^{\mathrm{a}}$ Kinetic energy of the explosion $\left(10^{51} \mathrm{erg} \mathrm{s}^{-1}\right)$

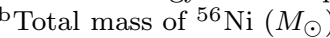

${ }^{c}$ Mass of $\mathrm{C}$ contained in the ejecta $\left(M_{\odot}\right)$

${ }^{\mathrm{d}}$ Mass of $\mathrm{O}$ contained in the ejecta $\left(M_{\odot}\right)$

${ }^{\mathrm{e}}$ Mass of Si contained in the ejecta $\left(M_{\odot}\right)$

${ }^{\mathrm{f}}$ Total mass of Fe group elements including ${ }^{56} \mathrm{Ni}\left(M_{\odot}\right)$

TABLE 6

Parameters of Spectral fitting

\begin{tabular}{ccccccccccc}
\hline \hline Epoch $^{\mathrm{a}}$ & $v_{\mathrm{ph}}{ }^{\mathrm{b}}$ & $M_{r, \mathrm{ph}}{ }^{\mathrm{c}}$ & $\log (L)^{\mathrm{d}}$ & $X(\mathrm{C})$ & $X(\mathrm{O})$ & $X(\mathrm{Si})$ & $X(\mathrm{~S})$ & $X(\mathrm{Ca})$ & $X(\mathrm{Fe})^{\mathrm{e}}$ & $\left.X^{(56} \mathrm{Ni}\right)^{\mathrm{f}}$ \\
\hline-6 & 6500 & 1.11 & 42.56 & $<0.001$ & 0.86 & 0.002 & 0.021 & 0.001 & 0.020 & 0.035 \\
+3 & 6000 & 1.05 & 42.66 & $<0.001$ & 0.77 & 0.020 & 0.002 & 0.001 & 0.020 & 0.14 \\
+14 & 3500 & 0.583 & 42.44 & $0.010(?)$ & 0.55 & 0.020 & 0.002 & 0.004 & 0.010 & 0.39 \\
+24 & 1500 & 0.128 & 42.19 & $0.010(?)$ & 0.53 & 0.020 & 0.022 & 0.004 & $0.40 \mathrm{~g}$ \\
+45 & 1000 & $4.90 \times 10^{-2}$ & 41.88 & - & 0.53 & 0.020 & 0.022 & 0.004 & $0.40 \mathrm{~g}$ \\
+228 & 250 & $1.18 \times 10^{-3}$ & 040.86 & - & 0.53 & 0.020 & 0.022 & 0.004 & $0.40 \mathrm{~g}$ \\
\hline
\end{tabular}

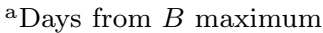

${ }^{\mathrm{b}}$ Photospheric velocity $\left(\mathrm{km} \mathrm{s}^{-1}\right)$

${ }^{\mathrm{c}}$ Mass coordinate at the photosphere $\left(M_{\odot}\right)$

${ }^{\mathrm{d}}$ Bolometric luminosity $\left(\mathrm{erg} \mathrm{s}^{-1}\right)$ in logarithm

${ }^{\mathrm{e}}$ Mass fraction of stable $\mathrm{Fe}$

${ }^{f}$ Mass fraction of radioactive ${ }^{56} \mathrm{Ni}$

${ }^{\mathrm{g}}$ The sum of stable Fe and ${ }^{56} \mathrm{Ni}$ 